DIVISION OF THE HUMANITIES AND SOCIAL SCIENCES

CALIFORNIA INSTITUTE OF TECHNOLOGY

PASADENA, CALIFORNIA 91125

FUNCTIONAL VOTING OPERATORS: THE NON-MONOTONIC CASE

Fuad Aleskerov

Institute of Control Sciences, Moscow

John Duggan

California Insitute of Technology

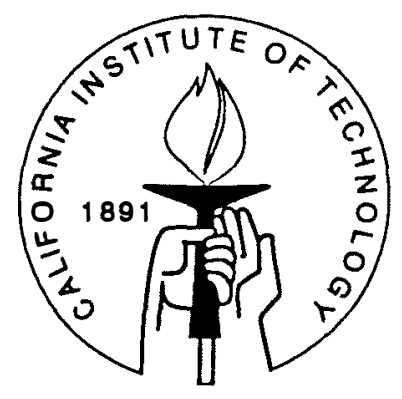

SOCIAL SCIENCE WORKING PAPER 858

August 1993 


\title{
FUNCTIONAL VOTING OPERATORS: THE NON-MONOTONIC CASE
}

\author{
Fuad Aleskerov John Duggan
}

\begin{abstract}
We extend the non-binary framework of social choice introduced by Aizerman and Aleskerov (1986), in which individual choice functions are aggregated into a social choice function, by considering non-monotonic operators. We characterize the class of "local" operators and provide the explicit forms of local operators satisfying various combinations of normative and rationality conditions in the absence of monotonicity. Surprisingly, the restriction of monotonicity is not binding for operators satisfying the usual rationality conditions. We identify two rationality restrictions which do admit non-monotonic operators. One restriction admits every sovereign and neutral operator, and the other admits only dictatorship and anti-dictatorship operators. This last result is a direct non-binary counterpart to Wilson's (1972) theorem.
\end{abstract}




\section{FUNCTIONAL VOTING OPERATORS: THE NON-MONOTONIC CASE*}

Fuad Aleskerov
John Duggan

In the classic social choice framework introduced by Arrow (1963), an individual's choice from any set of alternatives is given by a function which maximizes a weak order over the universe of alternatives, and social choices are likewise given by a function which maximizes a social weak order. Since the preference relation underlying individual or social choice can be recovered by presenting the decision-maker with choices over all pairs of alternatives, such choice functions are equivalent to the preference relations they maximize, and the aggregation of individual choice functions into a social choice function reduces to the mapping of individual orders into a social order. Such a mapping is a social welfare function. Arrow (1963) shows that the only social welfare functions satisfying non-imposedness, Independence of Irrelevant Alternatives, and monotonicity are dictatorial. ${ }^{1}$

In the more general, non-binary framework introduced by Aizerman and Aleskerov (1986), the aggregation of individual choice functions into a social choice function is performed by a functional voting operator, which does not require either individual or social choice functions to maximize a preference relation. As analysis of the class of all functional voting operators would be prohibitively difficult, they (as do we) restrict their attention to operators satisfying a locality condition similar in spirit to Arrow's Independence of Irrelevant Alternatives. For the class of local operators, Aizerman and Aleskerov characterize the operators satisfying monotonicity in conjunction with various other normative conditions. In addition, rationality of social choice is considered in the form of domain and range restrictions on operators, and it is shown that the local operators which satisfy minimal normative and rationality conditions are few or nonexistent.

*This paper was written during the first author's visit to the Division of the Humanities and Social Sciences at the California Institute of Technology. Partial financial support was received from the Caltech Laboratory for Experimental Economics and Political Science.

${ }^{1}$ Aleskerov and Vladmirov (1986) show that these conditions are equivalent to Arrow's original conditions. We use the former here only for rhetorical purposes. 
In the classic framework, Wilson (1972) and subsequently Aleskerov and Vladmirov (1986) replace monotonicity by the weaker condition of neutrality. They show that the only social welfare functions satisfying non-imposedness, Independence of Irrelevant Alternatives, and neutrality are dictatorial or inversely dictatorial: in particular, the only non-monotonic operators which satisfy these conditions are inversely dictatorial. In this paper, we extend the non-binary framework in Wilson's direction by completely characterizing the class of local operators-including non-monotonic operators-and considering the operators satisfying various combinations of normative and rationality conditions in the absence of monotonicity. Surprisingly, the restriction of monotonicity is not binding for operators satisfying "most" rationality conditions. That is, "most" domain and range restrictions are met only by monotonic operators. We identify two classes of operators isolated by domain and range restrictions that do admit non-monotonic operators. One class is defined on a very small domain and contains every sovereign and neutral operator, and the other class is defined on an intermediate domain and contains only two types of operators: dictatorships and anti-dictatorships. This last result is a direct non-binary counterpart to Wilson's theorem.

In Section 1, we introduce the non-binary framework in precise terms and outline the important results preceding this paper. We characterize the class of local operators in Appendix A and derive the explicit forms of all neutral operators. In Section 2, we state our results for local operators satisfying sovereignty and neutrality (with and without anonymity). In Section 3, we provide the explicit forms of local operators isolated by several rationality conditions in addition to sovereignty and neutrality (with and without anonymity), with proofs in Appendix B and Appendix C. And in Section 4, our results are discussed and the paper is concluded.

\section{The Non-Binary Framework}

Let $A$ be the finite universe of alternatives $\left\{x_{1}, \ldots, x_{m}\right\}$ and let $N$ be the finite population of individuals $\{1, \ldots, n\}$, where $m$ and $n$ are both integers greater than one. Assume that when presented with any admissible subset $X^{\prime} \in \mathcal{A}=2^{A} \backslash \emptyset$ each individual $i$ chooses a (possibly empty) set $C_{i}\left(X^{\prime}\right) \subseteq X^{\prime}$, where $x \in C_{1}\left(X^{\prime}\right)$ is to be interpreted as a vote for $x$ out of $X^{2}$ The collection of pairs $\left\{\left(C_{i}\left(X^{\prime}\right), X^{\prime}\right)\right\}_{X \in \mathcal{A}}$ entails a choice function $C_{i}: \mathcal{A} \rightarrow 2^{A}$, and the space of all such choice functions is denoted $\mathcal{C}$ with cross product $\mathcal{C}^{n}=\times_{i \in N} \mathcal{C}$.

The social choice function is determined by a functional voting operator $F: \mathcal{C}^{n} \rightarrow \mathcal{C}$ which takes every functional profile $\vec{C}=\left\{C_{i}\right\}_{i=1}^{n} \in \mathcal{C}^{n}$ of individual choice functions into a social choice function $F(\vec{C}) \in \mathcal{C}$. We will often write $C$ for $F(\vec{C}), C^{\prime}$ for $F\left(\vec{C}^{\prime}\right)$, and so on. Let $V(x, X ; \vec{C})=\left\{i \in N \mid x \in C_{\mathbf{i}}(X)\right\}$ be the set of individuals who choose $x$ out

\footnotetext{
${ }^{2}$ We will use the following convention for set inclusion: $\subseteq$ denotes weak inclusion, while $\subset$ is reserved for strict inclusion.
} 
of $X$. We restrict our analysis to operators which are local in the sense that the social choice of $x$ out of $X$ depends only on the set of individuals who vote for $x$ out of $X$ :

Definition 1 An operator $F$ is local if for all profiles $\vec{C}$ and $\vec{C}^{\prime}$

$$
\left[V(x, X ; \vec{C})=V\left(x, X ; \vec{C}^{\prime}\right)\right] \Rightarrow\left[x \in C(X) \Leftrightarrow x \in C^{\prime}(X)\right],
$$

for every admissible $X$ and $x \in X$. The class of local operators is denoted $\Lambda$.

This is clearly a strong restriction, excluding from our analysis many common methods of aggregation such as plurality rule and Borda rule.

There are several classes of local operators we will find particularly important:

Definition 2 An operator $F \in \Lambda$ is

(Un) a federation operator if there exists a collection $\Omega=\left\{\omega_{1}, \ldots, \omega_{q}\right\}$ with $q \geq 1$ and $\emptyset \subset \omega \subseteq N$ for all $\omega \in \Omega$ such that

$$
C(X)=\bigcup_{\omega \in \Omega} \bigcap_{i \in \omega} C_{i}(X)
$$

$(\cap \cup)$ a representation operator if there exists a collection $E=\left\{\epsilon_{1}, \ldots, \epsilon_{p}\right\}$ with $p \geq 1$ and $\emptyset \subset \epsilon \subseteq N$ for all $\epsilon \in E$ such that

$$
C(X)=\bigcap_{\epsilon \in E} \bigcup_{i \in \epsilon} C_{i}(X)
$$

( $\cap$ ) an oligarchy operator if there exists $\emptyset \subset \omega \subseteq N$ such that

$$
C\left(X^{\prime}\right)=\bigcap_{i \in \omega} C_{i}\left(X^{\prime}\right)
$$

(U) a syndicate operator if there exists $\emptyset \subset \epsilon \subseteq N$ such that

$$
C\left(X^{\prime}\right)=\bigcup_{i \in c} C_{i}(X)
$$

(d) a dictatorship operator if there exists $d \in N$ such that

$$
C(X)=C_{d}(X)
$$


(t) a $t$-majority operator if $1 \leq t \leq n, M=\{\mu \subseteq N|t \leq| \mu \mid\}$, and

$$
C(X)=\bigcup_{\mu \in M} \bigcap_{i \in \mu} C_{i}(X),
$$

for all admissible $X$. These classes are denoted $\Lambda^{\cup n}, \Lambda^{n \cup}, \Lambda^{n}, \Lambda^{\cup}, \Lambda^{d}$, and $\Lambda^{t}$, respectively.

Clearly, $t$-majority is the special case of federation operator with $\Omega=\{\omega \subseteq N|t \leq| \omega \mid\}$; oligarchy is the special case of federation with $q=1$; syndicate is the special case of representation with $p=1$; and dictatorship is the special case of both oligarchy and syndicate with $|\omega|=|\epsilon|=1$.

Also define the particular operators unanimity and varanimity, respectively, by

$$
\begin{aligned}
& F^{U}(\vec{C})(X)=\bigcap_{i \in N} C_{i}(X) \\
& F^{V}(\vec{C})(X)=\bigcup_{i \in N} C_{i}(X),
\end{aligned}
$$

for all admissible $X{ }^{3}$ Note that unanimity is the special case of oligarchy with $\omega=N$, and varanimity is the special case of syndicate with $\epsilon=N$. The following results are straightforward consequences of the above definitions:

\section{Theorem 1 (Aizerman and Aleskerov)}

$1 \Lambda^{\cup} \cup \Lambda^{n} \subset \Lambda^{\cup n}=\Lambda^{n \cup}$;

$2 \Lambda^{n} \cap \Lambda^{\cup}=\Lambda^{d}$;

$3 \Lambda^{t} \cap \Lambda^{d}=\emptyset$;

$4 \Lambda^{t} \cap \Lambda^{U}=\left\{F^{V}\right\}$;

$5 \Lambda^{t} \cap \Lambda^{n}=\left\{F^{U}\right\}$.

These relationships are represented by a Venn diagram in Figure 1.

(Figure 1 about here.)

We will explore the forms of local operators isolated by the following normative conditions and their combinations:

\footnotetext{
${ }^{3}$ Our exposition suffers for want of a widely accepted term for $F^{V}$. Aizerman and Aleskerov (1986) refer to this as "one-vote-for." This is unwieldly, however, and the term "varanimity" has the advantage of etymological correctness.
} 
Definition 3 An operator $F \in \Lambda$ satisfies

(S) sovereignty if for each admissible $X$ and $x \in X$, there is a profile $\vec{C}$ such that $x \in C(X)$. Moreover, for each admissible $X$ and $x \in X$, there is a profile $\vec{C}$ such that $x \notin C(X)$.

(M) monotonicity if for all profiles $\vec{C}$ and $\vec{C}^{\prime}$ and every admissible $X$ such that $x \in$ $C(X)$,

$$
\left[V(x, X ; \vec{C}) \subseteq V\left(x, X ; \vec{C}^{\prime}\right)\right] \Rightarrow\left[x \in C^{\prime}(X)\right] .
$$

(N) neutrality if for all profiles $\vec{C}$ and $\vec{C}^{\prime}$ and for all admissible $X$ and $X^{\prime}$ with $x \in X$ and $x^{\prime} \in X^{\prime}$,

$$
\left[V(x, X ; \vec{C})=V\left(x^{\prime}, X^{\prime} ; \vec{C}^{\prime}\right)\right] \Rightarrow\left[x \in C(X) \Leftrightarrow x^{\prime} \in C^{\prime}\left(X^{\prime}\right)\right] .
$$

(A) anonymity if for every permutation $\eta$ on $N$,

$$
F(\vec{C})=F\left(\left\{C_{\eta(i)}\right\}\right) .
$$

(P) Pareto if for each admissible $X, x \in C_{i}\left(X^{\prime}\right)$ for every individual implies $x \in C(X)$. Conversely, if for every individual $x \notin C_{\mathrm{i}}(X)$ then $x \notin C(X)$.

These classes are denoted $\Lambda^{S}, \Lambda^{M}, \Lambda^{N}, \Lambda^{A}$, and $\Lambda^{P}$, respectively.

We designate clases of operators satisfying combinations of these conditions by listing those conditions as superscripts: for example, $\Lambda^{S N}=\Lambda^{S} \cap \Lambda^{N}$. Note that monotonicity and neutrality imply locality, itself a strong restriction as mentioned above.

It is a simple matter to deduce the explicit forms of operators satisfying the first three and first four of these conditions:

\section{Theorem 2 (Aizerman and Aleskerov)}

$1 \Lambda^{S M N}=\Lambda^{\mathrm{Un}}=\Lambda^{\mathrm{nU}}$;

$2 \Lambda^{S M N A}=\Lambda^{t}$.

In addition to normative conditions, we are interested in isolating classes of operators by rationality conditions in the form of domain and range restrictions: 
Definition 4 The domain $Q_{d} \subseteq Q_{r} \subseteq \mathcal{C}$ is $Q_{r}$-closed by $F \in \Lambda$ if for all profiles $\vec{C} \in \times_{i \in N} Q_{d} F(\vec{C}) \in Q_{r}$. Let $\Lambda_{Q_{d}, Q_{r}}=\left\{F \in \Lambda \mid Q_{d}\right.$ is $Q_{r}$-closed by $\left.F\right\}$.

When $Q_{d}=Q_{r}=Q$, we denote $\Lambda_{Q_{d}, Q_{r}}$ simply by $\Lambda_{Q}$. The rationality conditions for operators are derived from the rationality conditions for choice functions:

Definition 5 A choice function $C$ satisfies

(H) heritage if

$$
\left[X_{1} \subseteq X_{2}\right] \Rightarrow\left[C\left(X_{2}\right) \cap X_{1} \subseteq C\left(X_{1}\right)\right]
$$

(C) concordance if

$$
C\left(X_{1}\right) \cap C\left(X_{2}\right) \subseteq C\left(X_{1} \cup X_{2}\right)
$$

(O) outcast if

$$
\left[X_{1} \subseteq X_{2} \backslash C\left(X_{2}\right)\right] \Rightarrow\left[C\left(X_{2} \backslash X_{1}\right)=C\left(X_{2}\right)\right]
$$

(K) constancy if

$$
\begin{aligned}
& {\left[X_{1} \subseteq X_{2}\right] \text { and }\left[C\left(X_{2}\right)=\emptyset \text { or } X_{1} \cap C\left(X_{2}\right) \neq \emptyset\right]} \\
& \quad \Rightarrow\left[C\left(X_{1}\right)=C\left(X_{2}\right) \cap X_{1}\right]
\end{aligned}
$$

for all admissible $X_{1}$ and $X_{2}$.

Let $(\mathbf{H})$ denote the condition of heritage, and let $\mathbf{H} \subset \mathcal{C}$ denote the set of choice functions that satisfy heritage. Likewise for $(\mathbf{C}),(\mathbf{O})$, and $(\mathbf{K}) \cdot{ }^{4}$ It is known that all eight intersections of combinations of $\mathbf{H}, \mathbf{C}$, and $\mathbf{O}$ and their complements $\overline{\mathbf{H}}, \overline{\mathbf{C}}$, and $\overline{\mathbf{O}}$ are non-empty, while $\mathbf{K} \subset \mathbf{H} \cap \mathbf{C} \cap \mathbf{O}$. Furthermore, a choice function satisfies $(\mathbf{K})$ if and only if it maximizes an extended weak order; ${ }^{5}$ a choice function satisfies $(\mathbf{H} \cap \mathbf{C} \cap \mathbf{O})$ if and only if it picks the undominated elements of an extended strict partial order; a choice function satisfies $(\mathbf{H} \cap \mathbf{O})$ if and only if it is path independent; ${ }^{6}$ and a choice

\footnotetext{
${ }^{4}$ The conditions $(\mathbf{H})$ and $(\mathbf{C})$ are called $\alpha$ and $\gamma$ by Sen $(1970) ;(\mathbf{O})$ is somewhat stronger than Sen's $\delta$; and $(\mathbf{K})$ was.originally introduced by Chernof (1954) and subsequently used by Arrow (1959). We use the notation of Aizerman and Malishevski (1981).

${ }^{5}$ An extended binary relation partitions the universe $A$ into $A_{1}$ and $A_{2}$, where the elements of $A_{1}$ are ordered by the binary relation and the elements of $A_{2}$ are dominated by each other and by the elements of $A_{1}$. This allows for the possibility of empty choice. A non-empty choice function satisfies $(\mathbf{K})$ if and only if it maximizes a weak order in the usual sense.

${ }^{6}$ These are the functions which satisfy Plott's (1973) condition of path independence: if $X=$ $\bigcup_{\psi \in \Psi} X_{\psi}=\bigcup_{\phi \in \Phi} X_{\phi}$ then $C\left(\bigcup_{\psi \in \Psi} C\left(X_{\psi}\right)\right)=C\left(\bigcup_{\phi \in \Phi} C\left(X_{\phi}\right)\right)$.
} 


\begin{tabular}{|l|c|c|c|c|}
\hline & $\Lambda_{\mathbf{H}}$ & $\Lambda_{\mathbf{C}}$ & $\Lambda_{\mathbf{O}}$ & $\Lambda_{\mathrm{HnC}}$ \\
\hline \hline$\Lambda^{S M N}$ & $\Lambda^{\mathrm{Un}}$ & $\Lambda^{n}$ & $\Lambda^{\mathrm{U}}$ & $\Lambda^{n}$ \\
\hline$\Lambda^{S M N A}$ & $\Lambda^{t}$ & $\left\{F^{U}\right\}$ & $\left\{F^{v}\right\}$ & $\left\{F^{U}\right\}$ \\
\hline \hline & $\Lambda_{\mathrm{HnO}}$ & $\Lambda_{\mathbf{C n O}}$ & $\Lambda_{\mathrm{H} \cap \mathrm{CnO}}$ & $\Lambda_{\mathbf{K}}$ \\
\hline \hline$\Lambda^{S M N}$ & $\Lambda^{\mathrm{U}}$ & $\Lambda^{d}$ & $\Lambda^{d}$ & $\Lambda^{d}$ \\
\hline$\Lambda^{S M N A}$ & $\left\{F^{v}\right\}$ & $\emptyset$ & $\emptyset$ & $\emptyset$ \\
\hline
\end{tabular}

Table 1: Theorem 3

function satisfies $(\mathbf{H} \cap \mathbf{C})$ if and only if it chooses the undominated elements of some binary relation. ${ }^{7}$

Operators from the class $\Lambda_{\mathbf{H}}$ are rational in the sense that the social choice function $F(\vec{C})$ inherits the rationality of the individual choice functions $C_{\boldsymbol{i}} \in \mathbf{H}$, and likewise for $\Lambda_{\mathbf{O}}, \Lambda_{\mathrm{H} \cap \mathbf{C}}$, and so on. An example of a class of operators isolated by rationality conditions are the social welfare functions: they are just $\Lambda_{\mathrm{K}}$. As Arrow first showed, the rationality conditions are extremely powerful in conjunction with the normative conditions:

Theorem 3 (Aizerman and Aleskerov) In Table 1, cells list the classes of operators satisfying the corresponding normative and rationality conditions.

\section{Non-Monotonic Operators: Normative Restric- tions}

The contribution of Aizerman and Aleskerov (1986) is an exhaustive analysis of the class $\Lambda^{M}$ of local monotonic operators, vielding Theorems 2 and 3 as special cases of more general theorems. Their results, however, leave untouched those local operators which fail monotonicity. In Appendix A, we characterize the class of local operators-including non-monotonic operators-and provide the explicit form of neutral operators satisfying sovereignty, anonymity, and their combinations. Here we present our results for the classes $\Lambda^{S N}$ and $\Lambda^{S N A}$, with proofs provided in Appendix A. Theorems 4 and 5 , below, are direct extensions of Theorems 1 and 2 .

\footnotetext{
${ }^{7}$ Note that these claims rely on our assumption that every pair of alternatives is an admissible set.
} 
An example of a non-monotonic operator is that defined by $C(X)=C_{i}(X) \cap C_{j}^{-}(X)$, where for a choice function $C$ we use $C^{-}$to denote the complement of $C: C^{-}(X)=$ $X \backslash C(X)$, for all admissible $X$. It does not follow, however, that an operator is nonmonotonic merely from the fact that it can be written as a union of intersections of choice functions with some "negative" terms: the operator defined by

$$
C(X)=\left[C_{1}(X) \cap C_{2}^{-}(X)\right] \cup\left[C_{1}^{-}(X) \cap C_{2}(X)\right] \cup\left[C_{1}(X) \cap C_{2}(X)\right]
$$

is actually monotonic since it can be rewritten as $C(X)=C_{1}(X) \cup C_{2}(X)$. Larger classes of operators which contain non-monotonic operators are introduced next:

Definition 6 An operator $F \in \Lambda$ is

$(\cup \cap \pm)$ a generalized federation operator if there exists a collection $\Omega=\left\{\omega_{1}, \ldots, \omega_{q}\right\}$ with $0<q<2^{n}$ such that

$$
C(X)=\bigcup_{\omega \in \Omega} \bigcap_{i \in N} C_{i}^{\omega}(X)
$$

where

$$
C_{i}^{\omega}(X)= \begin{cases}C_{i}(X) & \text { if } i \in \omega \\ C_{i}^{-}(X) & \text { else }\end{cases}
$$

$(\cap \cup \pm)$ a generalized representation operator if there exists a collection $E=\left\{\epsilon_{1}, \ldots, \epsilon_{p}\right\}$ with $0<p<2^{n}$ such that

$$
C\left(X^{\prime}\right)=\bigcap_{\epsilon \in E} \bigcup_{i \in N} C_{i}^{\epsilon}(X)
$$

where

$$
C_{i}^{\prime}= \begin{cases}C_{1}\left(X^{\prime}\right) & \text { if } i \in \epsilon \\ C_{1}^{-}\left(. X^{\prime}\right) & \text { else; }\end{cases}
$$

$\left(\cap^{-}\right)$an anti-oligarchy operator if there exists $\emptyset \subset \omega^{*} \subseteq N$ such that

$$
C(X)=\bigcap_{i \in \omega^{*}} C_{i}^{-}(X)
$$

$\left(\cup^{-}\right)$an anti-syndicate operator if there exists $\emptyset \subset \epsilon^{*} \subseteq N$ such that

$$
C(X)=\bigcup_{i \in \epsilon^{*}} C_{i}^{-}(X)
$$


$\left(d^{-}\right)$an anti-dictatorship operator if there exists $d \in N$ such that

$$
C(X)=C_{d}^{-}(X)
$$

( $T \pm$ ) a $T$-majority operator if $T$ is a non-empty set of integers between zero and $n$ with $T \neq\{0, \ldots, n\}, M=\{\mu \subseteq N|| \mu \mid \in T\}$, and

$$
C(X)=\bigcup_{\mu \in M} \bigcap_{i \in N} C_{i}^{\mu}(X)
$$

where

$$
C_{i}^{\mu}(X)= \begin{cases}C_{i}(X) & \text { if } i \in \mu \\ C_{i}^{-}(X) & \text { else }\end{cases}
$$

These classes are denoted $\Lambda^{\mathrm{\cup} \pm}, \Lambda^{\cap \cup \pm}, \Lambda^{\cap^{-}}, \Lambda^{\mathrm{U}^{-}}, \Lambda^{d^{-}}$, and $\Lambda^{T \pm}$, respectively.

It follows that $T$-majority is the special case of generalized federation with $\Omega=\{\omega \subseteq$ $N|| \omega \mid \in T\}$; an anti-oligarchy is the special case of generalized federation with $\Omega=\{\omega \subseteq$ $\left.N \mid \omega^{*} \cap \omega=\emptyset\right\}$; an anti-syndicate is the special case of generalized representation with $E=\left\{\epsilon \subseteq N \mid \epsilon^{*} \cap \epsilon=\emptyset\right\}$; and anti-dictatorship is the special case of both anti-oligarchy and anti-syndicate with $|\omega|=|\epsilon|=1$.

As an example of a generalized $T$-majority operator, let $n=5$ and $T=\{3,5\}$. Then an alternative $x$ is socially chosen from an admissible $X$ whenever exactly 3 or exactly 5 individuals vote for $x$-but if 1,2 , or 4 voters choose $x$.then it is socially rejected.

Also define the non-monotonic counterparts of unanimity and varanimity by

$$
\begin{aligned}
& F^{\ell^{\prime-}}(\vec{C})=\bigcap_{i \in N} C_{i}^{-}(X) \\
& F^{V^{\prime-}}(\vec{C})=\bigcup_{i \in N} C_{i}^{-}(X),
\end{aligned}
$$

for all admissible $X$. We call $F^{t^{1-}}$ negative unanimity since an alternative $x$ is chosen from $X$ if and only if it is chosen by no individual, and $F^{V^{\prime}}$ is called negative varanimity since an alternative $x$ is chosen from $X$ if and only if it is rejected by at least one individual. Negative unanimity is the special case of generalized federation and anti-oligarchy when $\Omega=\{\emptyset\}$ and it is the special case of $T$-majority when $T=\{0\}$. Negative varanimity is the special case of generalized representation and anti-syndicate when $E=\{\emptyset\}$ and it is the special case of $T$-majority when $T=\{0, \ldots, n-1\}$.

The next theorem extends Theorem 1 to the case of non-monotonic operators, revealing the structure of the class of generalized federation operators: 


\section{Theorem 4}

$1 \Lambda^{\cup \cap} \cup \Lambda^{U^{-}} \cup \Lambda^{n^{-}} \cup \Lambda^{T \pm} \subset \Lambda^{U \cap \pm}=\Lambda^{n \cup \pm}$;

$2 \Lambda^{U^{-}} \cap \Lambda^{n^{-}}=\Lambda^{d^{-}}$

$3 \Lambda^{n^{-}} \cap \Lambda^{\mathrm{Un}}=\Lambda^{\mathrm{U}^{-}} \cap \Lambda^{\mathrm{Un}}=\Lambda^{T \pm} \cap \Lambda^{d^{-}}=\emptyset$;

$4 \Lambda^{t} \subset \Lambda^{T \pm}$;

$5 \Lambda^{T \pm} \cap \Lambda^{U^{-}}=\left\{F^{V^{-}}\right\}$;

$6 \Lambda^{T \pm} \cap \Lambda^{n^{-}}=\left\{F^{U^{-}}\right\}$;

$7 \Lambda^{T \pm} \cap \Lambda^{\mathrm{Un}}=\Lambda^{t}$.

These relationships are represented by a Venn diagram in Figure 2.

(Figure 2 about here.)

We now extend Theorem 2 to the non-monotonic case. Together with Theorem 4, this provides the explicit form of all non-monotonic local operators isolated by sovereignty and neutrality:

\section{Theorem 5}

$1 \Lambda^{S N}=\Lambda^{\mathrm{Un \pm}}=\Lambda^{\cap \cup \pm}$;

$2 \Lambda^{S N A}=\Lambda^{T \pm}$.

\section{Non-Monotonic Operators: Rationality Restric- tions}

In this section, we consider the classes of local operators isolated by various rationality conditions combined with sovereignty and neutrality, with and without anonymity. Theorems 7 and 10 concern the properties of choice functions only and are proved in Appendix $\mathrm{C}$, while the other theorems in this section concern local operators and are proved in Appendix B. The first result is immediate, and extends Theorem 3 by adding two more rows to Table 1: 


\begin{tabular}{|l|c|c|c|c|}
\hline & $\Lambda_{\mathbf{H}}$ & $\Lambda_{\mathbf{C}}$ & $\Lambda_{\mathbf{O}}$ & $\Lambda_{\mathbf{H} \cap \mathbf{C}}$ \\
\hline \hline$\Lambda^{S N}$ & $\Lambda^{\mathrm{Un}}$ & $\Lambda^{n}$ & $\Lambda^{\mathrm{U}}$ & $\Lambda^{n}$ \\
\hline$\Lambda^{S N A}$ & $\Lambda^{t}$ & $\left\{F^{U}\right\}$ & $\left\{F^{V}\right\}$ & $\left\{F^{U}\right\}$ \\
\hline \hline & $\Lambda_{\mathbf{H} \cap \mathbf{O}}$ & $\Lambda_{\mathbf{C n O}}$ & $\Lambda_{\mathbf{H} \cap \mathbf{C} \cap \mathbf{O}}$ & $\Lambda_{\mathbf{K}}$ \\
\hline \hline$\Lambda^{S N}$ & $\Lambda^{\cup}$ & $\Lambda^{d}$ & $\Lambda^{d}$ & $\Lambda^{d}$ \\
\hline$\Lambda^{S N A}$ & $\left\{F^{V}\right\}$ & $\emptyset$ & $\emptyset$ & $\emptyset$ \\
\hline
\end{tabular}

Table 2: Theorem 6

Theorem 6 In Table 2, cells list the classes of operators satisfying the corresponding normative and rationality conditions.

Note that this is just the table of Theorem 3 with the rows appropriately relabeled! In other words, there are no non-monotonic operators which close these important domains. This is in stark contrast to the classic framework, in which Wilson (1972) and subsequently Aleskerov and Vladmirov (1986) show that the only non-dictatorial social welfare function satisfying appropriate formulations of locality, sovereignty, and neutrality are inversely dictatorial. This difference is partly accounted for by an attribute of operators in the classic framework which is not shared by operators in the non-binary framework: the inverse of a weak order is a weak order, but the complement of a choice function satisfying $(\mathbf{K})$ does not necessarily satisfy $(\mathbf{K})$.

Are there any interesting domains which are closed by non-monotonic operators? We next introduce several additional rationality conditions for operators in an attempt to answer this question. For a class $Q \subseteq \mathcal{C}$, let $Q^{-}=\left\{C \in \mathcal{C} \mid C^{-} \in Q\right\}$. That is, $Q^{-}$is the set of choice functions which are the complements of choice functions in $Q$. We then have the following result:

Theorem 7 The explicit forms of the conditions $\mathrm{H}^{-}, \mathrm{C}^{-}, \mathrm{O}^{-}$, and $\mathrm{K}^{-}$are

$\left(\mathrm{H}^{-}\right)\left[X_{1} \subseteq X_{2}\right] \Rightarrow\left[C\left(X_{1}^{\prime}\right) \subseteq C\left(X_{2}^{\prime}\right)\right]$

$\left(\mathrm{C}^{-}\right)\left[Y \subseteq X_{1} \cap X_{2}\right.$ and $\left.C\left(X_{1}\right) \cap Y^{\prime}=C\left(X_{2}\right) \cap Y^{\prime}=\emptyset\right]$

$\Rightarrow\left[C\left(X_{1} \cup X_{2}\right) \cap Y=\emptyset\right]$;

$\left(\mathbf{O}^{-}\right)\left[X_{1} \subseteq C\left(X_{2}\right)\right] \Rightarrow\left[C\left(X_{2} \backslash X_{1}\right)=C\left(X_{2}^{\prime}\right) \backslash X_{1}\right]$; 


\begin{tabular}{|c|c|c|c|c|}
\hline & $\overline{\Lambda_{\mathbf{H}^{-}}}$ & $\Lambda_{\mathbf{C}^{-}}$ & $\Lambda_{\mathbf{O}^{-}}$ & $\Lambda_{\mathbf{H}-n \mathbf{C}-}$ \\
\hline$\overline{\overline{\Lambda^{S N}}}$ & $\overline{\overline{\Lambda^{\mathrm{vn}}}}$ & $\overline{\overline{\Lambda^{U}}}$ & $\overline{\overline{\Lambda^{n}}}$ & 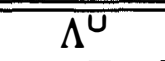 \\
\hline$\Lambda^{S N A}$ & $\Lambda^{t}$ & $\left\{F^{V}\right\}$ & $\left\{F^{U}\right\}$ & $\left\{F^{V}\right\}$ \\
\hline & $\overline{\Lambda_{\mathbf{H}-n O^{-}}}$ & $\overline{\mid \Lambda_{\mathbf{C}-n \mathbf{O}^{-}}}$ & $\Lambda_{\mathbf{H}-n C^{-n O}}$ & $\overline{\Lambda_{\mathrm{K}-}}$ \\
\hline$\overline{\Lambda^{S N}}$ & $\overline{\Lambda^{n}}$ & $\overline{\Lambda^{d}}$ & $\overline{\Lambda^{d}}$ & $\overline{\Lambda^{d}}$ \\
\hline$\Lambda^{S N A}$ & $\left\{F^{U}\right\}$ & $\theta$ & $\theta$ & $\theta$ \\
\hline
\end{tabular}

Table 3: Theorem 8

$\left(\mathrm{K}^{-}\right)\left[X_{1} \subseteq X_{2}\right]$ and $\left[X_{1} \nsubseteq C\left(X_{2}\right)\right.$ or $\left.C\left(X_{2}\right)=X_{2}\right]$

$\Rightarrow\left[C\left(X_{1}\right)=C\left(X_{2}\right) \cap X_{1}\right]$,

for all admissible $X_{1}, X_{2}$, and $Y$.

Note that $\mathbf{H}^{-}$is the domain of all monotonic choice functions, in the sense that if one set of alternatives is contained within another then the respective choice sets are in the same relation. For a choice function satisfying $\left(\mathbf{C}^{-}\right)$, if an element is rejected from two sets of alternatives then it is rejected from their union. For a choice function satisfying $\left(\mathrm{O}^{-}\right)$, a subset of chosen alternatives may be deleted without disturbing the choice set in any other way. Lastly, a choice function satisfying $\left(\mathbf{K}^{-}\right)$behaves much like one satisfying $(\mathbf{K})$, but it exhibits the peculiar property that the alternatives chosen from an admissible set are not necessarily chosen from the smaller admissible set consisting of just the originally chosen alternatives.

The classes of operators which close these new domains are investigated next:

Theorem 8 In Table 3, cells list the classes of operators satisfying the corresponding normative and rationality conditions.

Once again none of the eight domains is closed with respect to a non-monotonic operator.

One domain which is closed relative the entire class of generalized federation operators is defined next:

Definition 7 A choice function $C$ satisfies 
$\left(K^{*}\right)$ strong constancy if there exists $A^{*} \subseteq A$ such that

$$
C(X)=A^{*} \cap X,
$$

for all admissible $X$.

Choice over all admissible sets according to $\left(\mathbf{K}^{*}\right)$ is entirely given by the choice $A^{*}$ from the universe $A$. If $X$ is the set of alternatives then the alternatives chosen from $X$ are just the elements of $A^{*}$, and it follows that when $X \cap A^{*}=\emptyset$ the choice out of $X$ is empty. These choice functions are extremely rational, and it is easy to check that $\mathbf{K}^{*} \subseteq \mathbf{H} \cap \mathbf{H}^{-} \cap \mathbf{C} \cap \mathbf{C}^{-} \cap \mathbf{O} \cap \mathbf{O}^{-}$. This yields the following theorem:

Theorem $9 \Lambda_{\mathrm{K}^{*}} \cap \Lambda^{S N}=\Lambda^{\mathrm{un \pm}}$.

Although none of the sixteen domains considered in Theorems 6 and 8 is closed with respect to a non-monotonic operator, there are many other combinations which might

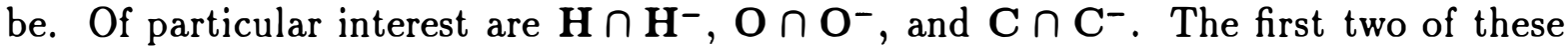
domains and their relationship to $\mathbf{K}^{*}$ is the subject of the next theorem and corollary:

Theorem $10 \mathrm{H} \cap \mathrm{H}^{-}=\mathrm{O} \cap \mathrm{O}^{-}=\mathrm{K}^{*}$.

Corollary $11 \Lambda_{\mathrm{H}_{\cap} \mathrm{H}^{-}} \cap \Lambda^{S N}=\Lambda_{\mathrm{O}^{-} \mathrm{O}^{-}} \cap \Lambda^{S N}=\Lambda^{\mathrm{Un \pm}}$.

We have then shown that there are no sovereign and neutral non-monotonic operators which close the usual domains, and that there is one extremely rational domain closed by every sovereign and neutral operator-non-monotonic as well as monotonic. The domain $\mathbf{C} \cap \mathbf{C}^{-}$is more interesting, as it does not demand the extreme rationality of $\mathbf{K}^{*}$ : for example, consider a choice function $C$ such that $C(\{x, y, z\})=\{x, y, z\}, C(\{x, y\})=\{x\}$, $C(\{y, z\})=\{y\}$, and $C(\{x, z\})=\{z\}$. This choice function satisfies $(\mathbf{C})$ and $\left(\mathbf{C}^{-}\right)$, but obviously violates $(\mathbf{H})$. Nevertheless. this domain is closed by exactly one monotonic operator and exactly one non-monotonic operator:

Theorem $12 \Lambda_{\mathrm{C} \cap \mathrm{C}^{-}} \cap \Lambda^{S N}=\Lambda^{d} \cup \Lambda^{d^{-}}$.

This is a direct non-binary counterpart to Wilson's theorem.

There are 63 non-empty domains isolated by combinations of the rationality conditions $(\mathbf{H}),(\mathbf{C}),(\mathbf{O}),\left(\mathbf{H}^{-}\right),\left(\mathbf{C}^{-}\right)$, and $\left(\mathbf{O}^{-}\right)$. Of these, 14 are covered by Theorems 6 and 
8 , and 28 reduce to the domain $\mathrm{K}^{*}$ and are covered by Theorem 9 . One more is treated in Theorem 13, leaving 20 domains which may be closed by a non-monotonic operator. Moreover, we have not considered the classes of operators which close domains which are strictly smaller than their ranges, such as $\Lambda_{\mathbf{H n C}, \mathbf{H}}$. It is possible to show, however, that of all these possibilities $\mathbf{C} \cap \mathbf{C}^{-}$is the only domain other than $\mathbf{K}^{*}$ which is closed by a non-monotonic operator.

\section{Conclusion}

We extend thë non-binary framework of Aizerman and Aleskerov (1986) by completely characterizing the class of local operators-monotonic and non-monotonic-and considering the operators satisfying various normative and rationality conditions in the absence of monotonicity. The explicit forms of all neutral operators are derived in Appendix $\mathrm{A}$, and these results are presented in Theorems 4 and 5 with the additional condition of sovereignty (with and without anyonymity). We also provide the explicit forms of sovereign and neutral operators which satisfy a range of rationality conditions.

This was done by Wilson (1972) in the classic framework, in which monotonicity is a binding constraint: when it is relaxed dictatorship and inverse dictatorship both satisfy the remaining conditions. In the non-binary framework, however, we find that monotonicity in conjunction with sovereignty, neutrality, and the usual rationality conditions is not binding. A more thorough investigation reveals that all sovereign and neutral operators-monotonic and non-monotonic-close the extremely rational domain $\mathbf{K}^{*}$, so that monotonicity is binding for such operators. Moreover, Wilson's theorem carries over to the non-binary framework in an extremely interesting way: there is one and only one domain $\mathbf{C} \cap \mathbf{C}^{-}$closed by just dictatorship and anti-dictatorship operators.

\section{A Proofs of Theorems: Normative Restrictions}

Define for every individual $i$ the boolean variable

$$
l_{i}(x, X)= \begin{cases}1 & \text { if } x \in C_{i}(X) \\ 0 & \text { else. }\end{cases}
$$

The locality of an operator $F$ is then equivalent to the existence of a boolean function $f(x, X ; \vec{l}(x, X))$ such that

$$
f(x, X ; \vec{l}(x, X))= \begin{cases}1 & \text { if } x \in C(X) \\ 0 & \text { else. }\end{cases}
$$


The main theorem of boolean logic shows that the function $f$ can be written explicitly in disjunctive normal form $\mathrm{as}^{8}$

$$
f(x, X ; \vec{l}(x, X))=\vee_{\omega \in \Omega(x, X)} \wedge_{i \in N} l_{i}^{\omega}(x, X),
$$

where $\Omega(x, X)=\left\{\omega_{1}^{(x, X)}, \ldots, \omega_{q(x, X)}^{(x, X)}\right\}$ is a collection of $q(x, X)$ sets of individuals varying with $x$ and $X$, and

$$
l_{i}^{\omega}(x, X)= \begin{cases}l_{i}(x, X) & \text { if } i \in \omega \\ \left|l_{i}(x, X)-1\right| & \text { else. }\end{cases}
$$

Alternatively, according to the theorem, $f$ can be written explicitly in conjunctive normal form as

$$
f(x, X ; \vec{l}(x, X))=\wedge_{\epsilon \in E(x, X)} \vee_{i \in N} l_{i}^{\epsilon}(x, X),
$$

where $E(x, X)=\left\{\epsilon_{1}^{(x, X)}, \ldots, \epsilon_{p(x, X)}^{(x, X)}\right\}$ and $l_{i}^{\epsilon}$ is defined in the same fashion as $l_{i}^{\omega}$.

It is easy to see that $x \in C(X)$ for all profiles when $\Omega(x, X)=2^{N}$. Not so obvious is the fact that for all profiles $x \notin C(X)$ when $E(x, X)=2^{N}$. To see this, suppose $x \in C(X)$, construct $\epsilon$ from $N$ by excluding the individuals who choose $x$ and including the individuals who reject $x$. By assumption, $\epsilon \in E(x, X)$. But since $x \in C(X)$, there must be either a member of $\epsilon$ who chooses $x$ or a non-member who rejects $x$, contradicting the construction of $\epsilon$. We adopt the conventions that summation over the empty set yields a value of zero and that multiplication over the empty set yields a value of one, so that for all profiles $x \notin C(X)$ when $\Omega(x, X)=\emptyset$ and $x \in C(X)$ when $E(x, X)=\emptyset$. Define the two trivial operators $F^{0}$ and $F^{1}$ by

$$
\begin{aligned}
& F^{0}(\vec{C})(X)=\emptyset \\
& F^{1}(\vec{C})(X)=X,
\end{aligned}
$$

for all admissible $X$. It then follows that $F=F^{0}$ when for all admissible $X$ and $x \in X$ we have $\Omega(x, X)=\emptyset$ or $E(x, X)=2^{N}$, and $F=F^{1}$ when for all admissible $X$ and $x \in X$ we have $\Omega(x, X)=2^{N}$ or $E(x, X)=\emptyset$.

The collection $\{\Omega(x, X)\}_{X \in \mathcal{A}, x \in X}$ is the disjunctive list for $F$, and it is a routine matter to check that for every profile it satisfies

$$
\left[x \in C\left(X^{\prime}\right)\right] \Leftrightarrow\left[I^{\prime}(x, X ; \vec{C}) \in \Omega(x, X)\right]
$$

for all admissible $X$. The collection $\left\{E\left(x, X^{\prime}\right)\right\}_{X \in \mathcal{A}, x \in X}$ is the conjunctive list for $F$, which for every profile-satisfies

$$
[x \notin C(X)] \Leftrightarrow[N \backslash V(x, X ; \vec{C}) \in E(x, X)],
$$

for all admissible $X$. To see this, assume $x \notin C(X)$, so that there is some $\epsilon \in E(x, X)$ such that all members of $\epsilon$ reject $x$ and all non-members choose $x$. But then we have $\epsilon=N \backslash V(x, X ; \vec{C}) \epsilon$ $E(x, X)$. Now assume $N \backslash V(x, X ; \vec{C}) \in E(x, X)$ and suppose that $x \in C(X)$. Then for every

\footnotetext{
${ }^{8} \vee$ and $\wedge$ are boolean addition and multiplication, respectively, defined by $l_{i} \vee l_{j}=1$ if $l_{i}+l_{j} \geq 1$ and $l_{i} \vee l_{j}=0$ otherwise, and $l_{i} \wedge l_{j}=1$ if $l_{i}+l_{j}=2$ and $l_{i} \wedge l_{j}=0$ otherwise.
} 
$\epsilon \in E(x, X)$ there is either a member who chooses $x$ or a non-member who rejects $x$, and in particular, this must be true for $N \backslash V(x, X ; \vec{C})$. This is, of course, a contradiction.

Since federation operators are monotonic, we can refine their disjunctive lists by omitting the listed $\omega$ 's which strictly contain another listed $\omega$. For example, the oligarchy operator given

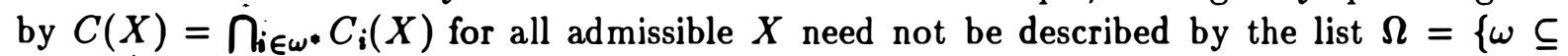
$\left.N \mid \omega^{*} \subseteq \omega\right\}$. Rather, the specification of $\omega^{*}$ itself is sufficient. For $F \in \Lambda^{\text {Un }}$ with disunctive list $\Omega$, define

$$
\Omega^{B}=\left\{\omega \in \Omega \mid \omega^{\prime} \not \subset \omega \text { for all } \omega^{\prime} \dot{\epsilon} \Omega\right\} .
$$

We call $\Omega^{B}$ the basic list for $F$, and naturally, we can similarly define basic lists $E^{B}$ for representation operators.

The above analysis characterizes the class $\Lambda$ of local operators in terms of boolean functions and in terms of lists. By reformulating the normative conditions in these terms, we characterize the classes of local operators satisfying them separately and in combination. In terms of disjunctive lists, sovereignty, monotonicity, neutrality, anonymity, and Pareto are

$\left(\mathbf{S}_{\omega}\right) \Omega(x, X) \neq \emptyset$ and $\Omega(x, X) \neq 2^{N}$;

$\left(\mathrm{M}_{\omega}\right)$ if $\omega \in \Omega(x, X)$ and $\omega \subseteq \omega^{\prime}$ then $\omega^{\prime} \in \Omega(x, X)$;

$\left(\mathrm{N}_{\omega}\right) \Omega(x, X)=\Omega\left(x^{\prime}, X^{\prime}\right)$ for all $X^{\prime}$ and $x^{\prime} \in X^{\prime}$;

$\left(\mathrm{A}_{\omega}\right)$ if $\omega \in \Omega(x, X)$ and $|\omega|=\left|\omega^{\prime}\right|$ then $\omega^{\prime} \in \Omega(x, X)$;

$\left(\mathbf{P}_{\omega}\right) \quad N \in \Omega(x, X)$ and $\emptyset \notin \Omega(x, X)$;

for all admissible $X$ and $x \in X$. Similar reformulations are possible in terms of boolean functions and conjunctive lists.

A sharper characterization is possible for the neutral classes $\Lambda^{N}, \Lambda^{S N}, \Lambda^{A N}$, and $\Lambda^{S N A}$. Neutrality is equivalent to the requirement that $\Omega(x, X)$ is independent of both $x$ and $X$, so that the disjunctive list for a neutral operator is simply $\Omega$. Define the replenished classes of operators:

$$
\begin{aligned}
& \tilde{\Lambda}^{\cup \cap \pm}=\Lambda^{\cup \cap \pm} \cup\left\{F^{0}, F^{1}\right\} \\
& \tilde{\Lambda}^{\cap U \pm}=\Lambda^{\cap \cup \pm} \cup\left\{F^{0}, F^{1}\right\} \\
& \tilde{\Lambda}^{T \pm}=\Lambda^{T \pm} \cup\left\{F^{0}, F^{1}\right\} .
\end{aligned}
$$

That is, we replenish $\Lambda^{\text {Un士 }}$ by allowing $q=0$ and $q=2^{n}$ in Definition 6 , so that $F \in \tilde{\Lambda}^{\text {un } \pm}$ if there exists a collection $\Omega=\left\{\omega_{1}, \ldots, \omega_{q}\right\}$ with $0 \leq q \leq 2^{n}$ such that $C(X)=\bigcup_{\omega \in \Omega} \bigcap_{i \in N} C_{i}^{\omega}(X)$. Similarly, we replenish $\Lambda^{\text {กUt }}$ by allowing $p=0$ and $p=2^{n}$, and we replenish $\Lambda^{T \pm}$ by allowing $T=\emptyset$ and $T=N \cup\{0\}$.

We then have the following general results: 
Theorem A.1 $\Lambda^{N}=\tilde{\Lambda}^{\mathrm{un} \pm}=\tilde{\Lambda}^{\mathrm{nu}}$.

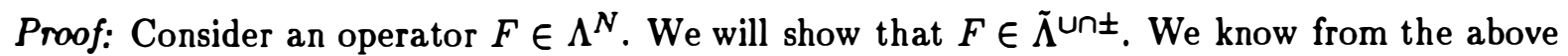
reformulation of neutrality in terms of boolean functions that $F$ can be represented by a function $f(\vec{l}(x, X))=\mathrm{V}_{\omega \in \Omega} \wedge_{i \in N} l_{i}^{\omega}(x, X)$, and we will show that $F(\vec{C})(X)=C(X)=\bigcup_{\omega \in \Omega} \bigcap_{i \in N} C_{i}^{\omega}(X)$ for every profile $\vec{C}$ and admissible $X$.

Assume that $x \in C(X)$ for some profile $\vec{C}$ and admissible $X$. Then there is an $\omega \in \Omega$ such that $V(x, X ; \vec{C})=\omega$. Then $x \in C_{i}(X)$ for all $i \in \omega$ and $x \in C_{j}^{-}(X)$ for all $j \notin \omega$, so $x \in \bigcap_{i \in N} C_{i}^{\omega}(X) \subseteq \bigcup_{\omega \in \Omega} \bigcap_{i \in N} C_{i}^{\omega}(X)$. Now assume that $x \in \bigcup_{\omega \in \Omega} \bigcap_{i \in N} C_{i}^{\omega}(X)$ for some profile $\vec{C}$ and admissible $X$. Then there is $\omega \in \Omega$ such that $x \in \bigcap_{i \in N} C_{i}^{\omega}(X)$, so $x \in C_{i}(X)$ for all $i \in \omega$ and $x \in C_{j}^{-}(X)$ for all $j \notin \omega$. That is, $V(x, X ; \vec{C})=\omega \in \Omega$, so $x \in C(X)$. Therefore, the desired equality holds and $F \in \tilde{\Lambda}^{\cup n \pm}$.

The proof that $F \in \tilde{\Lambda}^{n \cup \pm}$ is similar, using the conjunctive list for $F$. It is obvious that operators in replenished classes of generalized federation and generalized representation operators satisfy neutrality, giving us the desired equalities.

Theorem A.2 $\Lambda^{N A}=\tilde{\Lambda}^{T \pm}$.

Proof: From Theorem A.1, we know that $F \in \Lambda^{N A}$ satisfies $C(X)=\bigcup_{\omega \in \Omega} \bigcap_{i \in N} C_{i}^{\omega}(X)$ for some collection $\Omega$. By $\left(A_{\omega}\right)$, we know that if $\omega \in \Omega$ and $|\omega|=\left|\omega^{\prime}\right|$ then $\omega^{\prime} \in \Omega$. Now set $T=\{|\omega| \mid \omega \in \Omega\}$ and note that $F \in \tilde{\Lambda}^{T \pm}$. It is obvious that operators in the replenished class of $T$-majority operators satisfy neutrality and anonymity, giving us the desired equality.

Having characterized $\Lambda^{N}$ and $\Lambda^{N A}$, we next derive Theorem 5 , and once that is done the proof of Theorem 4 is straightforward:

\section{Theorem 5}

$1 \Lambda^{S N}=\Lambda^{\cup \cap \pm}=\Lambda^{\cap \cup \pm}$;

$2 \Lambda^{S N A}=\Lambda^{T \pm}$.

Proof: (1) Consider an operator $F \in \Lambda^{S N}$. From Theorem A.1, we know that $F \in \tilde{\Lambda}^{\text {Unt }}$, and by sovereignty it cannot be the case that $\Omega=\emptyset$ or $\Omega=2^{N}$. Then $\Omega=\left\{\omega_{1}, \ldots, \omega_{q}\right\}$ with $0<q<2^{n}$, and $F \in \Lambda^{\mathrm{Un} \pm}$. It is obvious that generalized federation operators satisfy sovereignty and neutrality. The proof that $\Lambda^{S N}=\Lambda^{n \cup \pm}$ is similar.

(2) Consider an operator $F \in \Lambda^{S N A}$. From Theorem A.2, we know that $F \in \tilde{\Lambda}^{T \pm}$, and by sovereignty it cannot be the case that $T=\emptyset$ or $T=\{0, \ldots, n\}$. But then $F \in \Lambda^{T \pm}$. It is obvious that $T$-majority operators satisfy sovereignty, neutrality, and anonymity. 


\section{Theorem 4}

$1 \Lambda^{\cup n} \cup \Lambda^{U^{-}} \cup \Lambda^{n^{-}} \cup \Lambda^{T \pm} \subset \Lambda^{U n \pm}=\Lambda^{n U \pm} ;$

$2 \Lambda^{U^{-}} \cap \Lambda^{n^{-}}=\Lambda^{d^{-}}$;

3 $\Lambda^{n^{-}} \cap \Lambda^{\cup r} \equiv \Lambda^{U^{-}} \cap \Lambda^{\cup n}=\Lambda^{T \pm} \cap \Lambda^{d^{-}}=\emptyset$;

$4 \Lambda^{t} \subset \Lambda^{T \pm}$

$5 \Lambda^{T \pm} \cap \Lambda^{U^{-}}=\left\{F^{V^{-}}\right\}$;

$6 \Lambda^{T \pm} \cap \Lambda^{n^{-}}=\left\{F^{U^{-}}\right\}$;

$7 \Lambda^{T \pm} \cap \Lambda^{\mathrm{Un}}=\Lambda^{t}$.

Proof: (1) It is shown in Theorem A.1 that $\Lambda^{\mathrm{Un} \pm}=\Lambda^{\mathrm{nU}}$. We know that $T$-majority, antioligarchy, and anti-syndicate are special cases of generalized federation and generalized representation, so we must show that $\Lambda^{\mathrm{Un}} \subseteq \Lambda^{\mathrm{un} \pm}$. Consider $F \in \Lambda^{\mathrm{Un}}$ with disjunctive list $\Omega$ and construct $\Omega^{\prime}$ from $\Omega$ by $\Omega^{\prime}=\left\{\omega^{\prime} \subseteq N \mid \omega \subseteq \omega^{\prime}\right.$ for some $\left.\omega \in \Omega\right\}$. Then for every profile $\vec{C}$ we have $C(X)=\bigcup_{\omega^{\prime} \in \Omega^{\prime}} \bigcap_{i \in N} C_{i}^{\omega}(X)$ for all admissible $X$ and $0<|\Omega|<2^{n}$, so that $F \in \Lambda^{\cup n \pm}$. To see that the inclusion is strict, consider the operator defined by $C(X)=C_{1}(X) \cap C_{2}^{-}(X)$ for all admissible $X$.

(2) Consider an operator $F \in \Lambda^{\mathrm{n}^{-}} \cap \Lambda^{\mathrm{U}^{-}}$. Since $F$ is an anti-syndicate operator, there is an individual $i$ who can secure the social choice of every alternative $x$ from every admissible $X$ by rejecting it. And since $F$ is an anti-oligarchy operator, there is an $\omega \subseteq N$ such that any member of $\omega$ can ensure that an alternative $x$ is socially rejected from an admissible $X$ by choosing it. It must be that $\{i\}=\omega$. But then $i$ is an anti-dictator and $F \in \Lambda^{d^{-}}$. The opposite inclusion is obvious, giving us the desired equality.

(3) It is clear that $\Lambda^{\mathrm{n}^{-}} \cap \Lambda^{\mathrm{U}}=\emptyset$, since anti-syndicate operators are non-monotonic and federation operators are monotonic. Similarly, we have $\Lambda^{\mathrm{U}^{-}} \cap \Lambda^{\mathrm{Un}}=\emptyset$. To see that $\Lambda^{T \pm} \cap \Lambda^{d^{-}}=$ $\emptyset$, suppose a $T$-majority operator is an anti-dictator operator. Then it must be that $T=\{1\}$, but then every voter would be an anti-dictator. This is, of course, impossible.

(4) For $F \in \Lambda^{t}$, set $T=\{t, \ldots, n\}$ and $M=\{\mu \subseteq N|| \mu \mid \in T\}$, and note that for every profile $\vec{C}$ we have $C(X)=\bigcup_{\mu \in \Lambda} \bigcap_{i \in N} C_{i}^{\mu}(X)$ for all admissible $X$, so that $F \in \Lambda^{T}$. To see that the inclusion .is strict, consider the example of $T$-majority in Section 2.

(5) Consider $F \in \Lambda^{T \pm} \cap \Lambda^{U^{-}}$. Since $F$ is an anti-syndicate operator, there is an $\epsilon$ such that every member of $\epsilon$ can secure the social choice of $x$ out of each admissible $X$ by rejecting it. Then it must be that $T=\{0, \ldots, n-1\}$, but then every voter can ensure the social choice of $x$ from $X$ by rejecting it. Therefore, $F=F^{v^{-}}$. The opposite inclusion is obvious, giving us the desired equality. 
(6) Consider $F \in \Lambda^{T \pm} \cap \Lambda^{n^{-}}$. Since $F$ is an anti-oligarchy operator, there is an $\omega \subseteq N$ such that $x$ is chosen out of each admissible $X$ if and only if $\omega \subseteq N \backslash V(x, X ; \vec{C})$. Then it must be that $T=\{0, \ldots, n-|\omega|\}$. Now if $|\omega|<n$ then we can find $i \in \omega$ and $j \notin \omega$, and construct $\omega^{\prime}$ by replacing $i$ with $j$, so that $|\omega|=\left|\omega^{\prime}\right|$. Since $F \in \Lambda^{T \pm}$ it follows that $x$ is chosen out of $X$ if $\omega^{\prime}=N \backslash V(x ; X ; \vec{C})$, but this is impossible because $i \in \omega \cap V(x, X ; \vec{C})$. Therefore, $|\omega|=n$ and $\dot{T}=\{0\}$. That is, $F=F^{U^{-}}$. The opposite inclusion is obvious, giving us the desired equality.

(7) Note that any operator $F \in \Lambda^{T \pm} \cap \Lambda^{u n}$ must be monotonic, so $T$ must be the set of all integers between some $t$ and $n$. Therefore, $F \in \Lambda^{t}$. The opposite inclusion is obvious, giving us the desired equality.

\section{B Proofs of Theorems: Rationality Restrictions}

Now that we have characterized the classes of local operators satisfying various normative restrictions, we turn to those classes isolated by rationality restrictions in conjunction with sovereignty and neutrality. Aizerman and Aleskerov (1986) provide a characterization in terms of disjunctive lists for the classes of operators that close the domains $\mathbf{H}, \mathbf{C}$, and $\mathbf{O}$, which we provide here for the special case of neutral operators:

$\left(\mathbf{H}_{\omega}\right)\left[\omega \in \Omega\right.$ and $\left.\omega \subseteq \omega^{\prime}\right] \Rightarrow\left[\omega^{\prime} \in \Omega\right]$;

$\left(\mathbf{C}_{\omega}\right)\left[\omega_{1} \in \Omega, \omega_{2} \in \Omega\right.$, and $\left.\omega_{1} \cap \omega_{2} \subseteq \omega\right] \Rightarrow[\omega \in \Omega]$;

$\left(\mathbf{O}_{\omega}\right)\left[\omega_{1} \in \Omega, \omega_{2} \notin \Omega\right.$, and $\left.\omega_{1} \backslash \omega_{2} \subseteq \omega \subseteq \omega_{1} \cup \omega_{2}\right] \Rightarrow[\omega \in \Omega]$,

and $\left[\omega_{2} \notin \Omega, \omega_{3} \notin \Omega\right.$, and $\left.\omega_{3} \backslash \omega_{2} \subseteq \omega \subseteq \omega_{3} \cup \omega_{2}\right] \Rightarrow[\omega \notin \Omega]$.

Moreover, these conditions carry over to two intersections of these domains:

$$
\begin{aligned}
& \Lambda_{\mathrm{H} \cap \mathrm{O}}=\Lambda_{\mathrm{H}} \cap \Lambda_{\mathrm{O}} \\
& \Lambda_{\mathrm{C} \cap \mathrm{O}}=\Lambda_{\mathrm{C}} \cap \Lambda_{\mathrm{O}} .
\end{aligned}
$$

That is, $F \in \Lambda_{\mathbf{H} \cap \mathbf{O}}$ if and only if it satisfies both conditions $\left(\mathbf{H}_{\omega}\right)$ and $\left(\mathbf{O}_{\omega}\right)$, and similarly for $\Lambda_{\mathrm{CnO}}$.

These results and the following lemma allow us to prove Theorem 6:

Lemma B.1 If $Q_{1} \subseteq Q_{2} \subseteq Q \subseteq \mathcal{C}$ then $\Lambda_{Q_{2}, Q} \subseteq \Lambda_{Q_{1}, Q}$. Furthermore, $\Lambda_{Q, Q_{1}} \subseteq \Lambda_{Q, Q_{2}}$.

Proof: Consider $F \in \Lambda_{Q_{2}, Q}$ and a profile $\vec{C} \in \mathrm{X}_{i \in N} Q_{1}$. Then since $\vec{C} \in \mathrm{x}_{i \in N} Q_{2}$ it immediately follows that $F(\vec{C}) \in Q$ and $F \in \Lambda_{Q_{1}, Q}$. Now consider $F \in \Lambda_{Q, Q_{1}}$ and a profile $\vec{C} \in \times_{i \in N} Q$. Then $F(\vec{C}) \in Q_{1} \subseteq Q_{2}$, and it follows that $F \in \Lambda_{Q, Q_{2}}$. 
Theorem 6 In Table 1, cells list the classes of operators satisfying the corresponding normative and rationality conditions.

Proof: The proof follows for columns $\Lambda_{\mathbf{H}}, \Lambda_{\mathbf{C}}, \Lambda_{\mathbf{O}}, \Lambda_{\mathbf{H} \cap \mathbf{O}}$, and $\Lambda_{\mathrm{CnO}}$ by noting that conditions $\left(\mathbf{H}_{\omega}\right)$ and $\left(\mathbf{C}_{\omega}\right)$ obviously imply the reformulation $\left(\mathbf{M}_{\omega}\right)$ of monotonicity in terms of disjunctive lists, so all of these classes are contained in $\Lambda^{M}$. That $\left(\mathbf{O}_{\omega}\right)$ also implies monotonicity can be seen by considering an operator $F \in \Lambda_{0}$ with $\omega^{\prime} \notin \Omega$. Setting $\omega_{2}=\omega_{3}=\omega^{\prime}$ in $\left(\mathbf{O}_{\omega}\right)$, it follows that $\left[\emptyset \subseteq \omega \subseteq \omega^{\prime}\right] \Rightarrow[\omega \notin \Omega]$, which is equivalent to $\left(\mathrm{M}_{\omega}\right)$.

Columns $\Lambda_{\mathbf{H}} \mathbf{C}, \Lambda_{\mathbf{H} \cap \mathbf{C} \cap \mathbf{O}}$, and $\Lambda_{\mathbf{K}}$ require a little more work. Since $\mathbf{K} \subseteq \mathbf{H} \cap \mathbf{C} \subseteq \mathbf{H} \cap \mathbf{C} \cap \mathbf{O}$, the desired testilt will follow from Lemma $B .1$ if we can show that $\Lambda_{K, \mathbf{H}} \subseteq \Lambda^{M}$. To this end, consider an operator $F \in \Lambda_{\mathrm{K}, \mathrm{H} \cap \mathrm{C}}$, assume that $\omega \in \Omega$, and $\omega \subseteq \omega^{\prime}$, and suppose that $\omega^{\prime} \notin \Omega$. Now construct the following profile for admissible $X_{1} \subseteq X_{2}$ and $x \in X_{1}$ :

$$
\begin{aligned}
& i \in N \backslash \omega^{\prime} \Rightarrow x \notin C_{i}\left(X_{1}\right) \cup C_{i}\left(X_{2}\right) \text {, } \\
& i \in \omega^{\prime} \backslash \omega \Rightarrow x \in C_{i}\left(X_{1}\right), C_{i}\left(X_{2}\right) \subseteq X_{2} \backslash X_{1} \text {, } \\
& i \in \omega \quad \Rightarrow \quad x \in C_{i}\left(X_{1}\right) \cap C_{i}\left(X_{2}\right) \text {, }
\end{aligned}
$$

and note that $C_{i}$ is consistent with $\mathbf{K}$ for every individual, so we can assume $\vec{C} \in \mathrm{x}_{i \in N} \mathbf{K}$. Furthermore, $V\left(x, X_{1} ; \vec{C}\right)=\omega^{\prime} \notin \Omega$ and $V\left(x, X_{2} ; \vec{C}\right)=\omega \in \Omega$. Then $x \in C\left(X_{2}\right)$ and $x \notin C\left(X_{1}\right)$, violating (H). Therefore, $\omega^{\prime} \in \Omega$ and $F$ is monotonic.

Theorem 8 is an easy consequence of Theorem 6 and the next two lemmas, in which $C^{1}$ denotes the choice function $C(X)=X$ for all admissible $X$ and $C^{\mathbf{0}}$ denotes the choice function $C(X)=\emptyset$ for all admissible $X$ :

Lemma B.2 For all $Q \subseteq \mathcal{C}, \Lambda^{\cap} \subseteq \Lambda_{Q}$ if and only if $\Lambda^{\cup} \subseteq \Lambda_{Q^{-}}$.

Proof: We prove only the "only if" direction, as the "if" direction follows by a similar argument. Assume that $\Lambda^{n} \subseteq \Lambda_{Q}$ and consider the operator $F \in \Lambda^{\cup}$, so there is $\omega \subseteq N$ such that for all profiles $\vec{C}$ we have $C(X)=\bigcup_{i \in \omega} C_{1}\left(X^{-}\right)$. for all admissible $X$. Pick a profile $\vec{C} \in x_{i \in N} Q^{-}$, relabel indexes so that $\omega=\{1, \ldots,|\omega|\}$, and for each $k \in \omega$ define the choice function $C(. ; k)$ by $C(X ; k)=\bigcup_{i=1}^{k} C_{i}(X)$ for all admissible $X . C(. ; 1)=C_{1} \in Q^{-}$follows immediately, so assume that $C(. ; k) \in Q^{-}$. Note that $C(. ; k+1)$ is defined by $C(X ; k+1)=C(X ; k) \cup C_{k+1}(X)$ for all admissible $X$. We know the operator defined by $C^{-}(X ; k+1)=C^{-}(X ; k) \cap C_{k+1}^{-}(X)$ belongs to $Q$ since $C^{-}(. ; k), C_{k+1} \in Q$ and $\Lambda^{n} \subseteq \Lambda_{Q}$. But then $C(. ; k+1) \in Q^{-}$, and by induction we have $C=C(. ;|\omega|) \in Q^{-}$. A similar argument proves the other direction.

Lemma B.3 For all $Q \subseteq \mathcal{C}$ such that $C^{\mathbf{1}}, C^{\mathbf{0}} \in Q$,

$1\left[\Lambda^{d} \subset \Lambda_{Q} \cap \Lambda^{\cup n}\right] \Rightarrow\left[\Lambda^{n} \subseteq \Lambda_{Q}\right.$ or $\left.\Lambda^{\cup} \subseteq \Lambda_{Q}\right]$ 
$2\left[\Lambda^{n} \subset \Lambda_{Q} \cap \Lambda^{\cup \cap}\right] \Rightarrow\left[\Lambda^{\cup n} \subseteq \Lambda_{Q}\right]$

$3\left[\Lambda^{\cup} \subset \Lambda_{Q} \cap \Lambda^{\cup n}\right] \Rightarrow\left[\Lambda^{\cup \cap} \subseteq \Lambda_{Q}\right]$.

Proof: (1) In words, we must show that if the dictatorship operators are strictly contained in the class of federation operators which close $Q$, then $Q$ is either closed with respect to intersection or union. Consider $F \in\left(\Lambda_{Q} \cap \Lambda^{\cup n}\right) \backslash \Lambda^{d}$. We know that there is a basic list $\Omega^{B}$ such that for every profile $\vec{C}$ we have $C(X)=\bigcup_{\omega \in \Omega B} \bigcap_{i \in \omega} C_{i}(X)$ for all admissible $X$. Since $F$ is not a dictatorship, we know that either $\left|\Omega^{B}\right|>1$ or $\Omega^{B}=\{\omega\}$ with $|\omega|>1$.

Assume the first case holds and define $\Omega_{i}=\left\{\omega \in \Omega^{B} \mid i \in \omega\right\}$ for every individual. We need two individuals $i$ and $j$ such that $\Omega_{i} \notin \Omega_{j}$ and $\Omega_{j} \notin \Omega_{i}$. If such a pair does not exist then for all pairs $\{i, j\} \subseteq N$ either $\Omega_{i} \subseteq \Omega_{j}$ or $\Omega_{j} \subseteq \Omega_{i}$, so we can order $N$ according to set inclusion of the $\Omega_{i}$ 's. Denote the set of maximal individuals by $N^{*}$, and note that $N^{*} \in \Omega^{B}$ and $N^{*} \subseteq \omega$ for all $\omega \in \Omega^{B}$. That is, $\Omega^{B}=\left\{N^{*}\right\}$ and $C(X)=\bigcap_{i \in N^{*}} C_{i}(X)$. Since $F$ is not a dictatorship we know that $\left|N^{*}\right|>1$, so $C(X)$ is the intersection of several choice functions from $Q$, and it follows that $\Lambda^{\cap} \subseteq \Lambda_{Q}$. To see this, suppose there are $C^{\prime}, C^{\prime \prime} \in Q$ such that $\hat{C} \notin Q$, where $\hat{C}(X)=C^{\prime}(X) \cap C^{\prime \prime}(X)$ for all admissible $X$. Pick $i \in N^{*}$ and consider the profile $\vec{C} \in \mathrm{x}_{i \in N} Q$ in which $C_{i}=C^{\prime}$ and $C_{j}=C^{\prime \prime}$ for $j \neq i$. Then $C=\hat{C} \notin Q$, contradicting the assumption that $F \in \Lambda_{Q}$.

So assume such a pair $\left\{i^{*}, j^{*}\right\}$ does exist and consider $\omega_{i} \in \in \Omega^{B}$ such that $i^{*} \in \omega_{i}$ and $j^{*} \notin \omega_{i^{*}}$ along with $\omega_{j^{*}} \in \Omega^{B}$ such that $j^{*} \in \omega_{j}$ and $i^{*} \notin \omega_{j^{*}}$. We will show that without loss of generality we can assume there is no $\omega \in \Omega^{B}$ such that $\omega \subseteq\left(\omega_{i} \bullet \cup \omega_{j} \cdot\right) \backslash\left\{i^{*}, j^{*}\right\}$. To see this, suppose there is such an $\omega$. Since $\Omega^{B}$ is a basic list, we know that $\omega \cap \omega_{i} * \neq \emptyset$ and $\omega \cap \omega_{j} \neq \emptyset$. Pick an individual $j \in \omega \cap \omega_{j}$ and relabel $j$ as $j^{*}$ and $\omega$ as $\omega_{j^{*}}$, and note that the new pair $\left\{i^{*}, j^{*}\right\}$ satisfies $\Omega_{i^{*}} \nsubseteq \Omega_{j^{*}}$ and $\Omega_{j} \bullet \nsubseteq \Omega_{i^{*}}$. This process will eventually terminate at a pair with the desired property.

Then consider the profile $\vec{C} \in \mathrm{x}_{i \in N^{N}} Q$ in which $C_{\boldsymbol{i}^{*}}$ and $C_{j}$. are arbitrary elements of $Q, C_{i}=$ $C^{1}$ for all other $i \in \omega_{i} \bullet \cup \omega_{j} \bullet$, and $C_{i}=C^{0}$ for all $i \notin \omega_{i} \bullet \cup \omega_{j} \bullet$. Then $C(X)=C_{i} \bullet(X) \cup C_{j} \bullet(X)$ for all admissible $X$, since $\bigcap_{i \in \omega_{\bullet}} C_{i}(X)=C_{\imath} \bullet(X), \bigcap_{i \in \omega, \bullet} C_{i}(X)=C_{j} \bullet(X)$, and

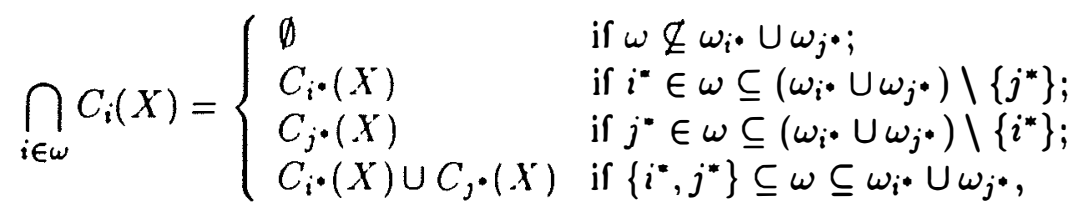

where we have dismissed without loss of generality the case $\omega \subseteq\left(\omega_{i} \bullet \cup \omega_{j^{*}}\right) \backslash\left\{i^{*}, j^{*}\right\}$. Since $F \in \Lambda_{Q}$ we know $C \in Q$, and it follows by an induction argument that $\Lambda^{\cup} \subseteq \Lambda_{Q}$.

If the first case does not hold then $\Omega^{B}=\{\omega\}$, where $|\omega|>1$. Then $F$ is just an oligarchy and it follows that $\Lambda^{n} \subseteq \Lambda_{Q}$.

(2) Consider $F \in\left(\Lambda_{Q} \cap \Lambda^{\cup n}\right) \backslash \Lambda^{n}$. We know that there is a basic list $\Omega^{B}$ such that for every profile $\vec{C}$ we have $C(X)=\bigcup_{\omega \in \Omega^{B}} \bigcap_{i \in \omega} C_{i}(X)$ for all admissible $X$. Since $F$ is not an 
oligarchy, we know that $\left|\Omega^{B}\right|>1$, which is just the first case in the proof of (1). Again, we need two individuals $i$ and $j$ such that $\Omega_{i} \nsubseteq \Omega_{j}$ and $\Omega_{j} \nsubseteq \Omega_{i}$. If such a pair does not exist then we know from the above argument that $F$ is an oligarchy with $\Omega^{B}=\left\{N^{*}\right\}$, a contradiction. It follows that we can find such a pair, and by the above argument we have $\Lambda^{\cup} \subseteq \Lambda_{Q}$. Since $Q$ is closed with respect to union and intersection, it follows that $\Lambda^{\mathrm{un}} \subseteq \Lambda_{Q}$.

(3) Consider $F \in\left(\Lambda_{Q} \cap \Lambda^{\cup n}\right) \backslash \Lambda^{\cup}$. We know that there is a basic list $\Omega^{B}$ such that for all profiles $C(X) \rightleftharpoons \bigcup_{\omega \in \Omega^{B}} \bigcap_{i \in \omega} C_{i}(X)$ for all admissible $X$. Since $F$ is not a syndicate, we know that there is $\omega^{\prime} \in \Omega^{B}$ with $\left|\omega^{\prime}\right|>1$, and since $\Omega^{B}$ is a basic list we know that there does not exist an $\omega \in \Omega^{B}$ with $\omega \subset \omega^{\prime}$.

Now consider the profile $\vec{C} \in \mathrm{X}_{i \in N} Q$ in which $C_{i} \in Q$ is arbitrary for every $i \in \omega^{\prime}$ and $C_{j}=C^{\mathbf{0}}$ for every $j \notin \omega^{\prime}$. Then $C(X)=\bigcap_{i \in \omega^{\prime}} C_{i}(X)$ for all admissible $X$, and since $F \in \Lambda_{Q}$ we have $C \in Q$. It follows by an induction argument that $\Lambda^{n} \subseteq \Lambda_{Q}$. Since $Q$ is closed with respect to union and intersection, it follows that $\Lambda^{\text {un }} \subseteq \Lambda_{Q}$.

Theorem 8 In Table 3, cells list the classes of operators satisfying the corresponding normative and rationality conditions.

Proof: The $\Lambda^{S N}$ row follows from the application of Theorem 6, Lemma B.2., and Lemma B.3, while the $\Lambda^{S N A}$ row then follows by application of the definition of anonymity. As an example, consider the cell $\Lambda^{S N} \cap \Lambda_{\mathbf{C}-}$. By Theorem $6, \Lambda^{n} \subseteq \Lambda_{\mathbf{C}}$, so it follows from Lemma B.2 that $\Lambda^{\cup} \subseteq \Lambda_{\mathbf{C}^{-}} \cap \Lambda^{\text {Un }}$. Now suppose this inclusion is strict, so that $\Lambda^{\cup} \subset \Lambda_{\mathbf{C}^{-}} \cap \Lambda^{\mathrm{Un}}$. Then by Lemma B.3 we have $\Lambda^{\cup n} \subseteq \Lambda_{\mathbf{C}^{-}}$, and in particular, $\Lambda^{n} \subseteq \Lambda_{\mathbf{C}^{-}}$. But then Lemma B.2 implies that $\Lambda^{\cup} \subseteq \Lambda_{\mathbf{C}}$, contradicting Theorem 6. Therefore, $\Lambda^{\cup}=\Lambda_{\mathbf{C}} \cap \cap \Lambda^{U n}=\Lambda_{\mathbf{C}}-\cap \Lambda^{S N}$. The results for the other cells follow by similar arguments.

The proof of Theorem 9 is quick work:

Theorem $9 \Lambda_{\mathrm{K}} \cdot \cap \Lambda^{S N}=\Lambda^{\mathrm{un} \pm}$

Proof: It suffices to show that $\mathrm{K}^{*}$ is closed with respect to union, intersection, and complementation. Consider $C_{1}, C_{2} \in \mathrm{K}^{*}$ given by $A_{i}$ and $A_{2}^{*}$, respectively, and consider the choice function $C$ defined by $C(X)=C_{1}\left(X^{*}\right) \cap C_{2}\left(X^{*}\right)$ for all admissible $X$. Since $x \in C(X)$ if and only if $x \in A^{*}=A_{1}^{*} \cap A_{2}^{*}$, it follows immediately that $C \in \mathrm{K}^{*}$. Similarly, the choice function $C$ defined by $C_{1}(X) \cup C_{2}(X)$ for all admissible $X$ satisfies $x \in C(X)$ if and only if $x \in A^{*}=A_{1}^{*} \cup A_{2}^{*}$, so $C \in \mathrm{K}^{*}$. Lastly, $C=C_{1}^{-} \in \mathrm{K}^{*}$, since $x \in C\left(X^{*}\right)$ if and only if $x \in A \backslash A_{1}^{*}$.

Theorem 12 is proved in a series of steps, in which we derive the explicit forms of operators in the classes $\Lambda_{\mathbf{C n C}^{-}, \mathbf{C}}$ and $\Lambda_{\mathbf{C n C} \mathbf{C}^{-}, \mathbf{C}^{-}}$and offer two corollaries of independent interest. In what follows, an interval is a collection of subsets of $N$ such as $\left[\omega^{\prime}, \omega^{\prime \prime}\right]=\left\{\omega \subseteq N \mid \omega^{\prime} \subseteq \omega \subseteq \omega^{\prime \prime}\right\}$. 
Theorem B:1 $F \in \Lambda_{\mathrm{CnC}-, \mathrm{C}} \cap \Lambda^{S N}$ if and only if $\Omega \neq\left\{2^{N}\right\}, \Omega \neq\{\emptyset\}$, and for all $\omega^{\prime}, \omega^{\prime \prime} \in \Omega$

$$
\left[\omega^{\prime} \cap \omega^{\prime \prime} \subseteq \omega \subseteq \omega^{\prime} \cup \omega^{\prime \prime}\right] \Rightarrow[\omega \in \Omega] .
$$

Proof: We first prove the "only if" direction. Consider $F \in \Lambda_{\mathrm{C} \cap \mathrm{C}-, \mathrm{C}} \cap \Lambda^{S N}$, so $F$ has a disjunctive list $\Omega$. It follows from sovereignty that $\Omega \neq\left\{2^{N}\right\}$ and $\Omega \neq\{\emptyset\}$. Suppose there are $\omega^{\prime}, \omega^{\prime \prime} \in \Omega$ and $\omega \subseteq N$ such that $\omega^{\prime} \cap \omega^{\prime \prime} \subseteq \omega \subseteq \omega^{\prime} \cup \omega^{\prime \prime}$ but $\omega \notin \Omega$. For $x \in X_{1} \cap X_{2}$ both admissible, construct the following profile $\overline{\vec{C}}$ :

$$
\begin{array}{lllll}
i \in N \backslash\left(\omega^{\prime} \cup \omega^{\prime \prime}\right) & \Rightarrow & x \notin C_{i}\left(X_{1}\right) & x \notin C_{i}\left(X_{2}\right) & x \notin C_{i}\left(X_{1} \cup X_{2}\right) \\
i \in \omega^{\prime} \backslash \omega & \Rightarrow & x \in C_{i}\left(X_{1}\right) & x \notin C_{i}\left(X_{2}\right) & x \notin C_{i}\left(X_{1} \cup X_{2}\right) \\
i \in \omega^{\prime \prime} \backslash \omega & \Rightarrow & x \notin C_{i}\left(X_{1}\right) & x \in C_{i}\left(X_{2}\right) & x \notin C_{i}\left(X_{1} \cup X_{2}\right) \\
i \in \omega \backslash \omega^{\prime} & \Rightarrow & x \notin C_{i}\left(X_{1}\right) & x \in C_{i}\left(X_{2}\right) & x \in C_{i}\left(X_{1} \cup X_{2}\right) \\
i \in \omega \backslash \omega^{\prime \prime} & \Rightarrow & x \in C_{i}\left(X_{1}\right) & x \notin C_{i}\left(X_{2}\right) & x \in C_{i}\left(X_{1} \cup X_{2}\right) \\
i \in \omega^{\prime} \cap \omega^{\prime \prime} & \Rightarrow & x \in C_{i}\left(X_{1}\right) & x \in C_{i}\left(X_{2}\right) & x \in C_{i}\left(X_{1} \cup X_{2}\right) .
\end{array}
$$

Note that this profile is consistent with $\mathbf{C}$ and $\mathbf{C}^{-}$, so we can assume $\vec{C} \in \mathrm{X}_{i \in N} \mathbf{C} \cap \mathbf{C}^{-}$. Now, since $V\left(x, X_{1} ; \vec{C}\right)=\omega^{\prime} \in \Omega$ we have $x \in C\left(X_{1}\right)$, and since $V\left(x, X_{2} ; \vec{C}\right)=\omega^{\prime \prime} \in \Omega$ we have $x \in C\left(X_{2}\right)$. But $V\left(x, X_{1} \cup X_{2} ; \vec{C}\right)=\omega$, so $x \notin C\left(X_{1} \cup X_{2}\right)$ and $C \notin \mathbf{C}$. This, of course, contradicts the assumption that $F \in \Lambda_{\mathrm{Cn}_{n-}, \mathrm{C}} \cap \Lambda^{S N}$.

We next prove the "if" direction. Consider an operator $F$ with disjunctive list $\Omega$ satisfying the above condition and a profile $\vec{C} \in \mathrm{x}_{i \in N} \mathbf{C} \cap \mathbf{C}^{-}$. Assume $x \in C\left(X_{1}\right) \cap C\left(X_{2}\right)$ for arbitrary admissible $X_{1}$ and $X_{2}$. Since $x \in C\left(X_{1}\right)$ we know $V\left(x, X_{1} ; \vec{C}\right) \in \Omega$, and since $x \in C\left(X_{2}\right)$ we know $V\left(x, X_{2} ; \vec{C}\right) \in \Omega$. The desired result will follow if it can be shown that

$$
V\left(x, X_{1} ; \vec{C}\right) \cap V\left(x, X_{2} ; \vec{C}\right) \subseteq V\left(x, X_{1} \cup X_{2} ; \vec{C}\right) \subseteq V\left(x, X_{1} ; \vec{C}\right) \cup V\left(x, X_{2} ; \vec{C}\right),
$$

for then the above list condition implies that $V\left(x, X_{1} \cup X_{2} ; \vec{C}\right) \in \Omega$ and $x \in C\left(X_{1} \cup X_{2}\right)$. Now, consider $i \in V\left(x, X_{1} ; \vec{C}\right) \cap V\left(x, X_{2} ; \vec{C}\right)$. which implies $x \in C_{i}\left(X_{1}\right) \cap C_{i}\left(X_{2}\right)$. Since $C_{i} \in \mathbf{C}$, it follows that $x \in C_{i}\left(X_{1} \cup X_{2}\right)$ and $i \in \mathrm{V}^{\prime}\left(x, X_{1} \cup X_{2} ; \vec{C}\right)$, giving us one inclusion. Consider $i \in V\left(x, X_{1} \cup X_{2} ; \vec{C}\right)$ and suppose $i \notin V^{\prime}\left(x, X_{1} ; \vec{C}\right) \cup V\left(x, X_{2} ; \vec{C}\right)$. Then $x \notin C_{i}\left(X_{1}\right)$ and $x \notin C_{i}\left(X_{2}\right)$, and it follows from Theorem $i$ and $C_{i} \in \mathrm{C}^{-}$that $x \notin C_{i}\left(X_{1} \cup X_{2}\right)$. In other words, $i \notin V\left(x, X_{1} \cup X_{2} ; \vec{C}\right)$, a contradiction. This gives us the second inclusion and the desired result.

Lemma B.4 $F \in \Lambda_{\mathrm{C} \cap \mathrm{C}^{-}, \mathrm{C}} \cap \Lambda^{S N}$ if and only if there is an interval $\left[\omega^{\prime}, \omega^{\prime \prime}\right] \neq\left\{2^{N}\right\}\left[\omega^{\prime}, \omega^{\prime \prime}\right] \neq$ $\{\emptyset\}$ such that for all profiles,

$$
C(X)=\bigcup_{\omega \in\left[\omega^{\prime}, \omega^{\prime \prime}\right]} \bigcap_{i \in N} C_{i}^{\omega}(X)
$$

for all admissible $X$. 
Proof: First the "only if" direction. Since $F \in \Lambda^{S N}=\Lambda^{\cup n \pm}$, we know from Theorem 5 that there is a collection $\Omega$ such that for all profiles $C(X)=\bigcup_{\omega \in \Omega} \bigcap_{i \in N} C_{i}^{\omega}(X)$. And since $F \in \Lambda_{\mathbf{C n C -}, \mathbf{C}}$, it follows from Theorem B.1 that $\Omega$ is an interval $\left[\omega^{\prime}, \omega^{\prime \prime}\right]$. To see this, note that Theorem B.1 implies $\bigcap_{\omega \in \Omega} \omega \in \Omega$ and $\bigcup_{\omega \in \Omega} \omega \in \Omega$. Then another application of Theorem B.1 implies $\left[\bigcap_{\Omega} \omega, \bigcup_{\Omega} \omega\right] \subseteq \Omega$. Equality then follows since $\Omega \subseteq\left[\bigcap_{\Omega} \omega, \bigcup_{\Omega} \omega\right]$. Since $F$ is sovereign, it must be that $\left[\omega^{\prime}, \omega^{\prime \prime}\right] \neq\left\{2^{N}\right\}$ and $\left[\omega^{\prime}, \omega^{\prime \prime}\right] \neq\{\emptyset\}$.

The "if" direction follows easily by noting that any operator with an interval disjunctive list is neutral and is also sovereign if the interval is not $\{\emptyset\}$ and not $\left\{2^{N}\right\}$. Furthermore, such an operator closes $\mathbf{C} \cap \mathbf{C}^{-}$since it satisfies the condition of Theorem B.1.

In words, Lemma B.4 establishes that there is an interval $\left[\omega^{\prime}, \omega^{\prime \eta}\right]$ such that an alternative $x$ is chosen from each admissible $X$ if and only if, for at least one group in the interval, all members choose $x$ from $X$ and all non-members reject $x$ from $X$.

Lemma B.5 $F \in \Lambda_{\mathrm{C} \cap \mathrm{C}-, \mathrm{C}-} \cap \Lambda^{S N}$ if and only if there is an interval $\left[\omega_{1}, \omega_{2}\right] \neq\left\{2^{N}\right\}$ and $\left[\omega_{1}, \omega_{2}\right] \neq\{\emptyset\}$ such that for all profiles,

$$
C(X)=\bigcap_{\omega \in\left[\omega_{1}, \omega_{2}\right]} \bigcup_{i \in N} C_{i}^{-\omega}(X),
$$

where

$$
C_{i}^{-\omega}(X)= \begin{cases}C_{i}^{-}(X) & \text { if } i \in \omega \\ C_{i}(X) & \text { else }\end{cases}
$$

for all admissible $X$.

Proof: We know that $F \in \Lambda_{\mathbf{C} \cap \mathbf{C}^{-}, \mathbf{C}^{-}} \cap \Lambda^{S N}$ if and only if $F \in \Lambda^{S N}$ and $F(\vec{C})=C \in \mathbf{C}^{-}$ for all profiles $\vec{C} \in \times_{i \in N} \mathbf{C} \cap \mathbf{C}^{-}$. This is true if and only if $F \in \Lambda^{S N}$ and for all profiles $\vec{C} \in \mathrm{X}_{i \in N} \mathbf{C} \cap \mathbf{C}^{-}$we have $C^{-} \in \mathbf{C}$, which by Lemma $\mathrm{B} .4$ is true if and only if there exists an interval $\left[\omega^{\prime}, \omega^{\prime \prime}\right] \neq\left\{2^{N}\right\}$ and $\left[\omega^{\prime}, \omega^{\prime \prime}\right] \neq\{\emptyset\}$ such that for all profiles

$$
C^{-}\left(X^{-}\right)=\bigcup_{\omega \in\left[\omega^{\prime}, \omega^{\prime \prime}\right]} \bigcap_{i \in N} C_{i}^{\omega}(X)
$$

for all admissible $X$. By DeMorgan's Law, this is true if and -only if there exists an interval $\left[\omega^{\prime}, \omega^{\prime \prime}\right] \neq\left\{2^{N}\right\}$ and $\left[\omega^{\prime}, \omega^{\prime \prime}\right] \neq\{\emptyset\}$ such that for all profiles

$$
C(X)=\bigcap_{\omega \in\left[\omega^{\prime}, \omega^{\prime \prime}\right]} \bigcup_{i \in N} C_{i}^{-\omega}(X),
$$

for all admissible $X$. The desired result follows by setting $\left[\omega_{1}, \omega_{2}\right]=\left[\omega^{\prime}, \omega^{\prime \prime}\right]$. 
In words, Lemma B.5 establishes that there is an interval $\left[\omega_{1}, \omega_{2}\right]$ such that an alternative $x$ is rejected from an admissible $X$ if and only if, for at least one group in the interval, all members choose $x$ from $X$ and all non-members reject $x$ from $X$.

These results allow us to prove two interesting corollaries, and after the proof of an additional lemma we can prove Theorem 13 as well:

Corollary B.2 $\Lambda^{n^{\prime}} \cup \Lambda^{n^{-}} \subset \Lambda_{\mathrm{C}_{n} \mathbf{C}^{-}, \mathrm{C}}$.

Proof: Note that an oligarchy operator $F$ is given by a disjunctive list $\Omega=\left\{\omega \subseteq N \mid \omega^{*} \subseteq \omega\right\}$ with $\emptyset \subset \omega^{*} \subseteq N$. This is just the interval $\left[\omega^{*}, N\right]$, and by Lemma B.4 it follows that $F \in \Lambda_{\mathrm{C} n \mathrm{C}-, \mathrm{C}}$.

We know that an anti-oligarchy operator $F$ is given by a disjunctive list $\Omega=\left\{\omega \subseteq N \mid \omega \cap \omega^{*}=\right.$ $\emptyset\}$ with $\emptyset \subset \omega^{*} \subseteq N$. This is just the interval $\left[\emptyset, N \backslash \omega^{*}\right]$, and by Lemma B.4 it follows that $F \in \Lambda_{\mathbf{C} \cap \mathbf{C}-, \mathbf{C}}$.

Corollary B.3 $\Lambda^{\cup} \cup \Lambda^{U^{-}} \subset \Lambda_{\mathrm{CnC}^{-}, \mathrm{C}^{-}}$.

Proof: Note that a syndicate operator $F$ is given by a conjunctive list $E=\left\{\epsilon \subseteq N \mid \epsilon^{*} \subseteq \epsilon\right\}$ with $\emptyset \subset \epsilon^{*} \subseteq N$. Then the interval $\left[\emptyset, N \backslash \epsilon^{*}\right]$ satisfies the condition of Lemma B.5 and it follows that $F \in \Lambda_{\mathrm{Cn} \mathrm{C}^{-}, \mathrm{C}^{-}}$.

We know that an anti-syndicate operator $F$ is given by a conjunctive list $E=\left\{\epsilon \subseteq N \mid \epsilon \cap \epsilon^{*}=\right.$ $\emptyset\}$ with $\emptyset \subset \epsilon^{*} \subseteq N$. Then the interval $\left[\epsilon^{*}, N\right]$ satisfies the condition of Lemma B.5 and it follows that $F \in \Lambda_{\mathrm{C}_{\cap} \mathrm{C}^{-}, \mathrm{C}^{-}}$.

Lemma B.6 $\Lambda_{\mathrm{C} \mathrm{C}^{-}, \mathrm{C}} \cap \Lambda_{\mathrm{C}_{\mathrm{C}} \mathrm{C}^{-}, \mathrm{C}^{-}}=\Lambda_{\mathrm{C}_{\mathrm{C}} \mathrm{C}^{-}} \cdot$

Proof: Consider $F \in \Lambda_{\mathbf{C}_{\cap} \mathbf{C}^{-}, \mathbf{C}} \cap \Lambda_{\mathbf{C n C}^{-}, \mathbf{C}^{-}}$and a profile $\vec{C} \in \times_{i \in N} \mathbf{C} \cap \mathbf{C}^{-}$. Then $F(\vec{C}) \in \mathbf{C}$ and $F(\vec{C}) \in \mathbf{C}^{-}$, so $F \in \Lambda_{\mathbf{C} \cap \mathbf{C}^{-}}$. Conversely, $F \in \Lambda_{\mathbf{C} \cap \mathbf{C}^{-}}$maps each profile in $\mathbf{C} \cap \mathbf{C}^{-}$to $\mathbf{C}$ and $\mathbf{C}^{-}$, so $F \in \Lambda_{\mathbf{C}_{\cap} \mathbf{C}^{-}, \mathbf{C}} \cap \Lambda_{\mathbf{C}_{n} \mathbf{C}^{-}, \mathbf{C}^{-}} \cdot$

Theorem $13 \Lambda_{\mathrm{C} \cap \mathrm{C}^{-}} \cap \Lambda^{S N}=\Lambda^{d} \cup \Lambda^{d^{-}}$.

Proof: Obviously, $\mathbf{C} \cap \mathbf{C}^{-}$is closed with respect to complementation, so we have $\Lambda^{d} \cup \Lambda^{d^{-}} \subseteq$ $\Lambda_{\mathrm{C} \cap \mathrm{C}}-\cap \Lambda^{S N}$.

Now consider $F \in \Lambda_{\mathbf{C} \cap \mathbf{C}^{-}} \cap \Lambda^{S N}$. By Lemma B.6, we know that $F \in \Lambda_{\mathbf{C} \cap \mathbf{C}^{-}, \mathbf{C}} \cap \Lambda_{\mathbf{C}_{n} \mathbf{C}^{-}, \mathbf{C}^{-}}$. Lemma B.4 then implies that $F$ is given by an interval $\left[\omega^{\prime}, \omega^{\prime \prime}\right]$ such that for every profile $\vec{C}$ an 
alternative $x$ is socially chosen from each admissible $X$ if and only if $V(x, X ; \vec{C}) \in\left[\omega^{\prime}, \omega^{\prime}\right]$. And Lemma B.5 implies that $F$ is given by an interval $\left[\omega_{1}, \omega_{2}\right]$ such that $x$ is socially rejected from each admissible $X$ if and only if $V(x, X ; \vec{C}) \in\left[\omega_{1}, \omega_{2}\right]$. It follows immediately that $\left[\omega^{\prime}, \omega^{\prime \prime}\right]=2^{N} \backslash\left[\omega_{1}, \omega_{2}\right]$ and $\left[\omega^{\prime}, \omega^{\prime \prime}\right] \cap\left[\omega_{1}, \omega_{2}\right]=\emptyset$. Also from Lemma B.4 we have $\left[\omega^{\prime}, \omega^{\prime \prime}\right] \neq[\emptyset, N]$, $\left[\omega^{\prime}, \omega^{\prime \prime}\right] \neq \emptyset$, and similarly for $\left[\omega_{1}, \omega_{2}\right]$. Note that $F \in \Lambda^{d}$ if and only if there is a $d \in N$ such that $\left[\omega_{1}, \omega_{2}\right]=[\theta, N \backslash\{d\}]$ and $F \in \Lambda^{d^{-}}$if and only if there is a $d \in N$ such that $\left[\omega_{1}, \omega_{2}\right]=[\{d\}, N]$.

Suppose that $F \notin \Lambda^{d}$ and $F \notin \Lambda^{d^{-}}$. We consider three cases:

1 there is a $d \in N$ such that $d \in \omega$ for all $\omega \in\left[\omega_{1}, \omega_{2}\right]$;

2 there is a $d \hat{\epsilon} N$ such that $d \notin \omega$ for all $\omega \in\left[\omega_{1}, \omega_{2}\right]$;

3 for all $i \in N$ there are $\omega_{i}^{1}, \omega_{i}^{0} \in\left[\omega_{1}, \omega_{2}\right]$ such that $i \in \omega_{i}^{1}$ and $i \notin \omega_{i}^{0}$.

We shall see that a contradiction arises in each case, giving us the desired result.

(1) Since $\left[\omega_{1}, \omega_{2}\right] \neq[\{d\}, N]$, there exists an $\omega^{d} \subseteq N$ with $d \in \omega^{d}$ such that $\omega^{d} \notin\left[\omega_{1}, \omega_{2}\right]$. Note that we then have $[\emptyset, N \backslash\{d\}] \cup\left\{\omega^{d}\right\} \subseteq\left[\omega^{\prime}, \omega^{\prime \prime}\right]$. In particular, $N \backslash \omega^{d} \in\left[\omega^{\prime}, \omega^{\prime \prime}\right]$. It follows from Theorem B.1 that $\omega^{d} \cap\left(N \backslash \omega^{d}\right)=\emptyset \in\left[\omega^{\prime}, \omega^{\prime \prime}\right]$ and that $\omega^{d} \cup\left(N \backslash \omega^{d}\right)=N \in\left[\omega^{\prime}, \omega^{\prime \prime}\right]$. But then $\left[\omega^{\prime}, \omega^{\prime \prime}\right]=[\emptyset, N]$, violating sovereignty.

(2) Since $\left[\omega_{1}, \omega_{2}\right] \neq[\emptyset, N \backslash\{d\}]$, there exists an $\omega^{d} \subseteq N$ with $d \notin \omega^{d}$ such that $\omega^{d} \notin\left[\omega_{1}, \omega_{2}\right]$. Note that we then have $[\{d\}, N] \cup\left\{\omega^{d}\right\} \subseteq\left[\omega^{\prime}, \omega^{\prime}\right]$. It follows from Theorem B.1 that $\omega^{d} \cap\{d\}=$ $\emptyset \in\left[\omega^{\prime}, \omega^{\prime}\right]$. But then $\left[\omega^{\prime}, \omega^{\prime \prime}\right]=[\emptyset, N]$, violating sovereignty.

(3) Since $\left[\omega_{1}, \omega_{2}\right]$ is an interval, we know $\bigcap_{i \in N} \omega_{i}^{0}=\emptyset \in\left[\omega_{1}, \omega_{2}\right]$ and $\bigcup_{i \in N} \omega_{i}^{1}=N \in\left[\omega_{1}, \omega_{2}\right]$. But then $\left[\omega_{1}, \omega_{2}\right]=[\emptyset, N]$, violating sovereignty.

\section{Proofs of Theorems: Choice Functions}

Theorem 7 The explicit forms of the conditions $\mathrm{H}^{-}, \mathrm{C}^{-}, \mathrm{O}^{-}$, and $\mathrm{K}^{-}$are

$\left(\mathbf{H}^{-}\right)\left[X_{1} \subseteq X_{2}\right] \Rightarrow\left[C\left(X_{1}\right) \subseteq C\left(X_{2}\right)\right]$;

$\left(\mathrm{C}^{-}\right)\left[Y \subseteq X_{1} \cap X_{2}\right.$ and $\left.C\left(X_{1}\right) \cap Y=C\left(X_{2}\right) \cap Y=\emptyset\right]$

$\Rightarrow\left[C\left(X_{1} \cup X_{2}\right) \cap Y=\emptyset\right]$;

$\left(\mathbf{O}^{-}\right)\left[X_{1} \subseteq C\left(X_{2}\right)\right] \Rightarrow\left[C\left(X_{2} \backslash X_{1}\right)=C\left(X_{2}\right) \backslash X_{1}\right]$;

$\left(\mathrm{K}^{-}\right)\left[X_{1} \subseteq X_{2}\right]$ and $\left[X_{1} \nsubseteq C\left(X_{2}\right)\right.$ or $\left.C\left(X_{2}\right)=X_{2}\right] \Rightarrow\left[C\left(X_{1}\right)=C\left(X_{2}\right) \cap X_{1}\right]$,

for all admissible $X_{1}, X_{2}$, and $Y$. 
Proof: $\left(\mathbf{H}^{-}\right)$Consider admissible $X_{1} \subseteq X_{2}, C \in \mathbf{H}^{-}$, and $x \in C\left(X_{1}\right)$. Then $x \notin C^{-}\left(X_{1}\right)$ and since $C^{-} \in \mathbf{H}$, it follows that $x \notin C^{-}\left(X_{2}\right)$. Therefore, $x \in C\left(X_{2}\right)$.

Now consider admissible $X_{1} \subseteq X_{2}$ and $C$ satisfying the above condition. We know that $C\left(X_{1}\right) \subseteq C\left(X_{2}\right)$, so $C^{-}\left(X_{2}\right)=X_{2} \backslash C\left(X_{2}\right) \subseteq X_{2} \backslash C\left(X_{1}\right)$. Then $C^{-}\left(X_{2}\right) \cap X_{1} \subseteq\left(X_{2} \backslash C\left(X_{1}\right)\right) \cap$ $X_{1}=X_{1} \backslash C\left(X_{1}\right)=C^{-}\left(X_{1}\right)$. Therefore, $C^{-} \in \mathbf{H}$ and $C \in \mathbf{H}^{-}$.

$\left(\mathbf{C}^{-}\right)$Consider $C \in \mathbf{C}^{-}, Y \subseteq X_{1} \cap X_{2}$ admissible, and $C\left(X_{1}\right) \cap Y=C\left(X_{2}\right) \cap Y=\emptyset$. Since $Y \subseteq X_{1}$ and $C\left(X_{1}\right) \cap Y=\emptyset$, we know $Y \subseteq C^{-}\left(X_{1}\right)$. Similarly, $Y \subseteq C^{-}\left(X_{2}\right)$. Since $C^{-} \in \mathbf{C}$, it follows that $Y \subseteq C^{-}\left(X_{1}\right) \cap C^{-}\left(X_{2}\right) \subseteq C^{-}\left(X_{1} \cup X_{2}\right)$. Therefore, $C\left(X_{1} \cup X_{2}\right) \cap Y=\emptyset$.

Now consider $C$ satisfying the above condition. Let $x \in C^{-}\left(X_{1}\right) \cap C^{-}\left(X_{2}\right)$. Then $\{x\} \subseteq$ $X_{1} \cap X_{2}$ and $C\left(X_{1}\right) \cap\{x\}=C\left(X_{2}\right) \cap\{x\}=\emptyset$, so $C\left(X_{1} \cup X_{2}\right) \cap\{x\}=\emptyset$. We then have $\{x\} \subseteq C^{-}\left(X_{1} \cup X_{2}\right)$. Therefore, $C^{-} \in \mathbf{C}$ and $C \in \mathbf{C}^{-}$.

$\left(\mathbf{O}^{-}\right)$Consider $C \in \mathrm{O}^{-}$and admissible $X_{1} \subseteq C\left(X_{2}\right)$. Then $X_{1} \subseteq X_{2} \backslash C^{-}\left(X_{2}\right)$ and $C^{-}\left(X_{2} \backslash\right.$ $\left.X_{1}\right)=C^{-}\left(X_{2}\right)$, since $C^{-} \in \mathbf{O}$. This implies $\left(X_{1} \backslash X_{2}\right) \backslash C^{-}\left(X_{2} \backslash X_{1}\right)=\left(X_{2} \backslash X_{1}\right) \backslash C^{-}\left(X_{2}\right)$, which implies $C\left(X_{2} \backslash X_{1}\right)=\left(X_{2} \backslash C^{-}\left(X_{2}\right)\right) \backslash X_{1}=C\left(X_{2}\right) \backslash X_{1}$.

Now consider $C$ satisfying the above condition and admissible $X_{1} \subseteq X_{2} \backslash C^{-}\left(X_{2}\right)$. Then $X_{1} \subseteq C\left(X_{2}\right)$, so we have $C\left(X_{2} \backslash X_{1}\right)=C\left(X_{2}\right) \backslash X_{1}$, which implies $\left(X_{2} \backslash X_{1}\right) \backslash C\left(X_{2} \backslash X_{1}\right)=$ $\left(X_{2} \backslash X_{1}\right) \backslash\left(C\left(X_{2}\right) \backslash X_{1}\right)$. This in turn implies $C^{-}\left(X_{2} \backslash X_{1}\right)=\left[\left(X_{2} \backslash X_{1}\right) \backslash C\left(X_{2}\right)\right] \cup\left[C\left(X_{2}\right) \cap\right.$ $\left.X_{1} \cap\left(X_{2} \backslash X_{1}\right)\right]=\left[\left(X_{2} \backslash C\left(X_{2}\right)\right) \backslash X_{1}\right]=\left[C^{-}\left(X_{2}\right) \backslash X_{1}\right]=C^{-}\left(X_{2}\right)$. Therefore, $C^{-} \in \mathbf{O}$ and $C \in \mathbf{O}^{-}$.

$\left(\mathrm{K}^{-}\right)$Consider $C \in \mathrm{K}^{-}$and admissible $X_{1} \subseteq X_{2}$ with $C\left(X_{2}\right)=X_{2}$ or $X_{1} \& C\left(X_{2}\right)$. First assume $C\left(X_{2}\right)=X_{2}$. Then $C^{-}\left(X_{2}\right)=\emptyset$, where $C^{-} \in \mathbf{K}$. It follows that $C^{-}\left(X_{1}\right)=$ $C^{-}\left(X_{2}\right) \cap X_{1}=\emptyset$, so $C\left(X_{1}\right)=X_{1}=C\left(X_{2}\right) \cap X_{1}$.

Next assume $X_{1} \notin C\left(X_{2}\right)$, and suppose that $X_{1} \cap C^{-}\left(X_{2}\right)=X_{1} \cap\left(X_{2} \backslash C\left(X_{2}\right)\right)=\emptyset$. But then $X_{1} \subseteq C\left(X_{2}\right)$, a contradiction. So $X_{1} \cap C^{-}\left(X_{2}\right) \neq \emptyset$, and since $C^{-} \in \mathrm{K}$ we have $C^{-}\left(X_{1}\right)=C^{-}\left(X_{2}\right) \cap X_{1}$, which implies $X_{1} \backslash C^{-}\left(X_{1}\right)=X_{1} \backslash\left(C^{-}\left(X_{2}\right) \cap X_{1}\right)$. This in turn implies $C\left(X_{1}\right)=X_{1} \backslash C^{-}\left(X_{2}\right)=X_{1} \backslash\left(X_{2} \backslash C\left(X_{2}\right)\right)=\left(X_{1} \backslash X_{2}\right) \cup\left(X_{1} \cap X_{2} \cap C\left(X_{2}\right)\right)=X_{1} \cap C\left(X_{2}\right)$.

Now consider $C$ satisfying the above condition and $C^{-}\left(X_{2}\right)=\emptyset$ or $X_{1} \cap C^{-}\left(X_{2}\right) \neq \emptyset$ for admissible $X_{1} \subseteq X_{2}$. First assume $C^{-}\left(X_{2}\right)=\emptyset$. Then $C\left(X_{2}\right)=X_{2}$, so $C\left(X_{1}\right)=C\left(X_{2}\right) \cap X_{1}=$ $X_{1}$, which implies $C^{-}\left(X_{1}\right)=\emptyset=X_{1} \cap C^{-}\left(X_{2}\right)$.

Next assume $X_{1} \cap C^{-}\left(X_{2}\right) \neq \emptyset$. But then $X_{1} \cap\left(X_{2} \backslash C\left(X_{2}\right)=X_{1} \backslash C\left(X_{2}\right)=X_{1} \backslash\left(X_{2} \backslash\right.\right.$ $\left.C^{-}\left(X_{2}\right)\right)=\left(X_{1} \backslash X_{1}\right) \cup\left(X_{1} \cap X_{2} \cap C^{-}\left(X_{2}\right)\right)=X_{1} \cap C^{-}\left(X_{2}\right) \neq \emptyset$, so $X_{1} \& C\left(X_{2}\right)$. Then $C\left(X_{1}\right)=C\left(X_{2}\right) \cap X_{1}$, which implies $X_{1} \backslash C\left(X_{1}\right)=X_{1} \backslash\left(C\left(X_{2}\right) \cap X_{1}\right)$. This in turn implies $C^{-}\left(X_{1}\right)=\left(X_{1} \backslash C\left(X_{2}\right)\right) \cup\left(X_{1} \backslash X_{1}\right)=X_{1} \backslash\left(X_{2} \backslash C^{-}\left(X_{2}\right)\right)=\left(X_{1} \backslash X_{2}\right) \cup\left(X_{1} \cap X_{2} \cap C^{-}\left(X_{2}\right)\right)=$ $X_{1} \cap C^{-}\left(X_{2}\right)$. Therefore, $C^{-} \in \mathrm{K}$ and $C \in \mathrm{K}^{-}$.

Theorem $10 \mathrm{H} \cap \mathrm{H}^{-}=\mathrm{O} \cap \mathrm{O}^{-}=\mathrm{K}^{*}$. 
Proof: Consider $C \in \mathrm{H} \cap \mathrm{H}^{-}$and set $A^{*}=C(A)$. Then for every admissible $X$ we have $A^{*} \cap X \subseteq C(X)$ and $C(X) \subseteq A^{*} \cap X$, which implies $C(X)=A^{*} \cap X$ and $C \in \mathrm{K}^{*}$. That is, $\mathbf{H} \cap \mathbf{H}^{-} \subseteq \mathbf{K}^{*}$. It is obvious that $\mathbf{K}^{*} \subseteq \mathbf{H} \cap \mathbf{H}^{-}$, so equality follows.

Consider $C \in \mathbf{O} \cap \mathbf{O}^{-}$and set $A^{*}=C(A)$. For an admissible $X$, partition $A \backslash X$ into $X_{1}=(A \backslash X) \cap A^{*}$ and $X_{2}=(A \backslash X) \backslash A^{*}$. Note that $X_{1} \subseteq A^{*}$ and $X_{2} \subseteq\left(A \backslash X_{1}\right) \backslash\left(A^{*} \backslash X_{1}\right)$. To see this last inclusion, note that $X_{2}=(A \backslash X) \backslash A^{*} \subseteq A \backslash A^{*}=\left(A \backslash X_{1}\right) \backslash A^{*}=\left(A \backslash X_{1}\right) \backslash\left(A^{*} \backslash X_{1}\right)$. Then $C\left(A \backslash X_{1}\right)=A^{*} \backslash X_{1}$ and $C\left(\left(A \backslash X_{1}\right) \backslash X_{2}\right)=\left(A^{*} \backslash X_{1}\right) \backslash X_{2}=A^{*} \cap X$. But $X=\left(A \backslash X_{1}\right) \backslash X_{2}$, so we have $C(X)=A^{*} \cap X$. That is, $\mathbf{O} \cap \mathbf{O}^{-} \subseteq \mathbf{K}^{*}$. It is obvious that $\mathbf{K}^{*} \subseteq \mathbf{O} \cap \mathbf{O}^{-}$, so equality follows. 


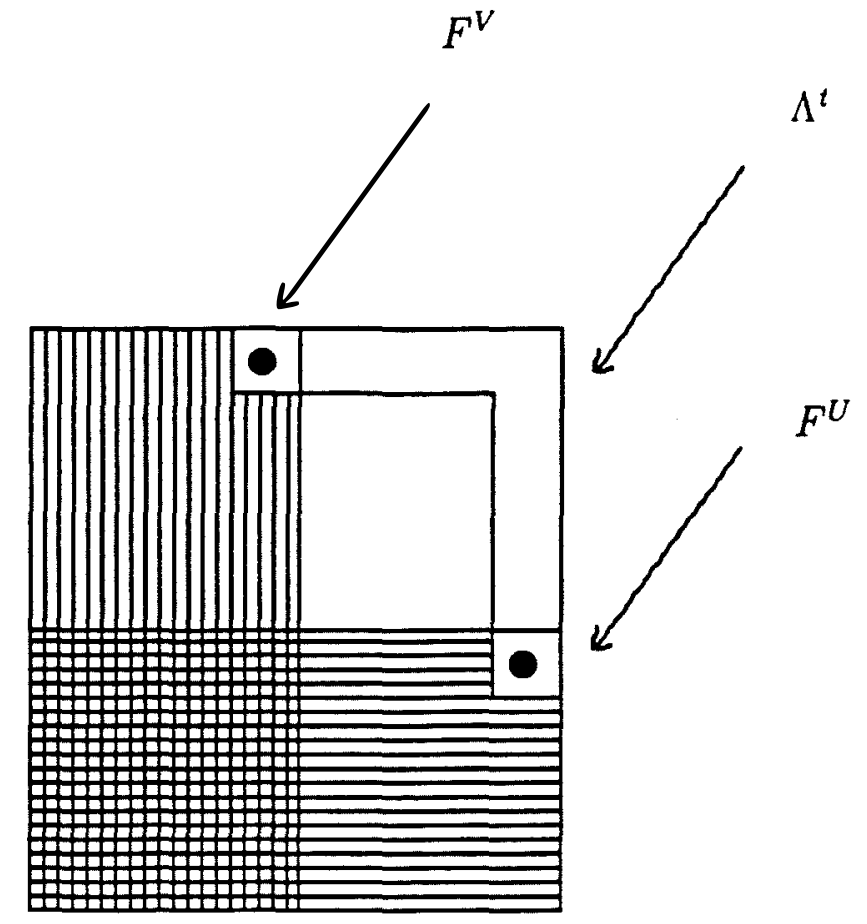

$\Lambda^{\text {un }}$

川ाI $\Lambda^{u}$

E $\Lambda^{n}$

$\# \Lambda^{d}$

Figure 1: Theorem 1 


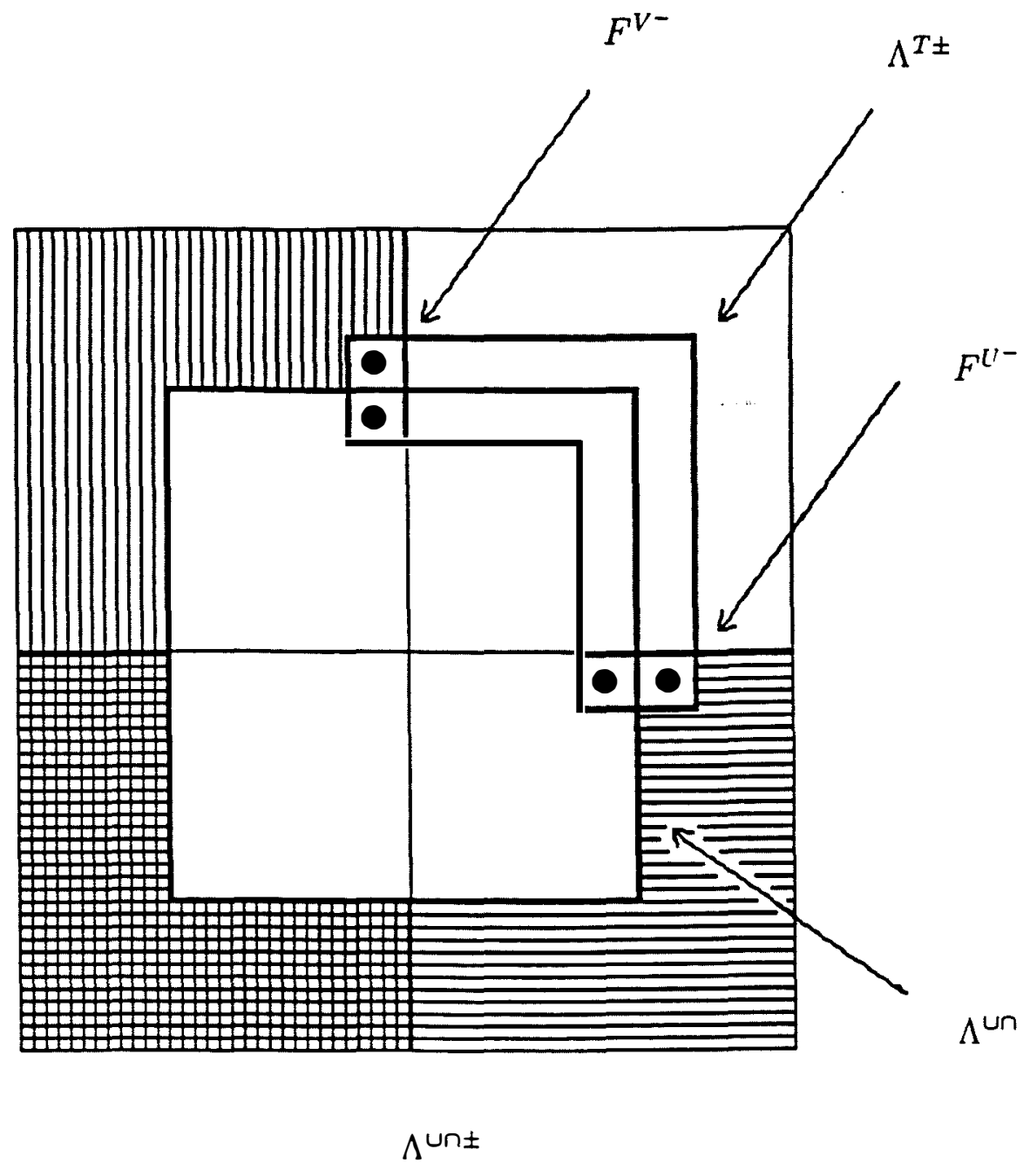

$\Lambda^{u^{-}}$
$\equiv \Lambda^{n^{-}}$
$\Lambda^{d^{-}}$

Figure 2: Theorem 4 


\section{References}

[1] M. A. Aizerman and F. T. Aleskerov, Voting operators in the space of choice functions, Math. Soc. Sci. 11 (1986) 201-242.

[2] M. A. Aizerman and A. V. Malishevski, General theory of best variants choice: some aspects, IEEE Transactions on Automatic Control AC-26(5) (1981) 1030-1041.

[3] F. T. Aleskerov and A. V. Vladmirov, Hierarchical voting, Info. Sci. 39 (1986) 41-86.

[4] K. J. Arrow, Rational choice functions and orderings, Econometrica 26 (1959) 121-127.

[5] K. J. Arrow, Social Choice and Individual Values, 2nd ed. (Wiley, New York, 1963).

[6] H. Chernoff, Rational selection of decision functions, Econometrica 22 (1954) 422-443.

[7] C. R. Plott, Path independence, rationality, and social choice, Econometrica 41 (1973) 1075-1091.

[8] A. K. Sen, Collective Choice and Social Welfare, (Holden-Day, San Francisco, 1970).

[9] R. Wilson, Social choice theory without the Pareto principle, J. of Econom. Theory 5 (1972) 478-486. 